\title{
Das Recht auf Leben und Gesundheit, Patentschutz und das Verfahren des High Court of South Africa zur Verfassungsmäßigkeit des südafrikanischen Medicines and Related Substances Control Amendment Act
}

\author{
Von Jörg Fedtke, Hamburg
}

\section{Einführung}

Vor dem High Court of South Africa in Pretoria ist am 19. April 2001 die juristische Auseinandersetzung um geplante Änderungen des südafrikanischen Patentrechts mit einem Vergleich zwischen der klagenden Pharmaceutical Manufacturers Association of South Africa (PMA) sowie 39 pharmazeutischen Unternehmen und der südafrikanischen Regierung beendet worden. ${ }^{1}$ In der Sache ging es bei diesem weltweit beachteten Verfahren in erster Linie um die Verfassungsmäßigkeit des Medicines and Related Substances Control Amendment Act, ${ }^{2}$ einem Gesetz zur Änderung des Arzneimittelgesetzes. ${ }^{3}$ Die Novelle, die nach der vergleichsweisen Rücknahme der Klage seitens der Pharmaindustrie mit einer Verzögerung von über drei Jahren in Kraft treten konnte, erleichtert insbesondere die Einfuhr und Produktion preiswerter Nachahmungspräparate patentgeschützter Arzneimittel entgegen den Bestimmungen des südafrikanischen Patentgesetzes. ${ }^{4}$

Das Vorgehen der Pharmakonzerne in Südafrika ist überwiegend als Klage gegen preisgünstige Aids-Medikamente verstanden worden, ${ }^{5}$ obwohl sich die angefochtenen Regelungen des Amendment Act nicht auf Medikamente gegen die Immunschwäche Aids (aquired immune deficiency syndrome) beschränken und daher auch Arzneimittel zur Behandlung etwa von Tuberkulose oder Malaria erfassen. Richtig ist allerdings, dass die Aids-Katastro-

High Court of South Africa (Transvaal Provincial Division), Case No. 4183/98 (sog. „AIDSVerfahren").

Act 90 of 1997 .

3 Medicines and Related Substances Control Act, No. 101 of 1965 (Medicines Act).

4 Patents Act, No. 57 of 1978.

5 Vgl. etwa folgende Presseberichte über das Verfahren: Guardian Unlimited, London, vom 5.3.2001 (http://www.guardianunlimited.co.uk/international/story/0,3604,446465,00.html); The Washington Post, Washington, D.C., vom 6.3.2001 (http://www.washingtonpost.com/wpdyn/articles/ A26902-2001Mar5.html); Frankfurter Rundschau, Frankfurt a.M., vom 20.4.2001, S. 1 . 
phe im südlichen Afrika die Änderungen des Arzneimittelrechts initiiert hat. Allein in der Republik Südafrika sind gegenwärtig zwischen 4 und 6 Millionen Menschen infiziert (etwa $10 \%$ der Bevölkerung), wobei Experten derzeit von 1.700 neuen Infektionen pro Tag ausgehen. Jährlich sterben schätzungsweise 250.000 Südafrikaner an Aids; betroffen ist dabei hauptsächlich die erwachsene, ökonomisch aktive Bevölkerung im Alter von 20 bis 40 Jahren, die Familien ernährt und die wirtschaftliche Entwicklung des Landes trägt. ${ }^{6}$ Als Hauptursachen der hohen Verbreitungsrate gelten mangelnde Aufklärung, die schlechte medizinische Grundversorgung (vielerorts sind erst Investitionen in das Gesundheitswesen nötig, da Aids-Medikamente unter ständiger Betreuung nach einem strengen Behandlungsplan eingenommen werden müssen), Aberglaube, die häufig anzutreffende Tabuisierung sexueller Themen in traditionellen afrikanischen Gesellschaften und promiskuitives Sexualverhalten. ${ }^{7}$ Zudem hat die zweite frei gewählte Regierung Südafrikas den Kampf gegen die Seuche nur langsam aufgenommen; ${ }^{8}$ noch anlässlich der 13 . Welt-Aids-Konferenz im vergangenen Jahr wurde der Zusammenhang zwischen der HIV-Infektion und Aids von Staatspräsident Thabo Mbeki in Frage gestellt. ${ }^{9}$ Aufklärung tut aber gerade in ländlichen Gebieten und Schulen Not - viele Infizierte wissen beispielsweise gar nicht, dass sie überhaupt Virus-Träger sind. Inzwischen hat das südafrikanische Gesundheitsministerium eine große Kampagne gestartet, um der Situation Herr zu werden. ${ }^{10}$ Gesundheitsministerin Manto Tschabalala-Msimang (ANC) baut dabei auch auf die Änderungen des Arzneimittelrechts, um die medizinische Versorgung im Land zu verbessern.

Zur Situation in Südafrika vgl. Unaids / WHO, Epidemiological Fact Sheet on HIV/AIDS and sexually transmitted infections - South Africa 2000 update - (zu finden über die Homepage des Joint United Nations Programme on HIV/AIDS (Unaids) unter http://www.unaids.org in der Rubrik ,HIV/AIDS info by country'). Zum Vergleich: In ganz Westeuropa geht die UN von 540.000 HIV-Infizierten aus. Nach Angaben des Berliner Robert-Koch-Instituts gibt es in Deutschland heute zwischen 30.000 und 40.000 HIV-Infizierte und 5.000 bis 6.000 Menschen mit Aids. Jährlich infizieren sich hierzulande etwa 2.000 Personen neu (vgl. Frankfurter Rundschau vom 29.6.2001, S. 5). Die Zahl der weltweit erkrankten Menschen wird von Gesundheitsexperten auf 36 Millionen geschätzt. Etwa 53 Millionen Menschen sind HIV-infiziert; die tägliche Infektionsrate liegt bei 15.000. Zwischen 18 und 22 Millionen Menschen sind bereits an Aids gestorben. Zur Verbreitung von Aids vgl. die folgenden weiteren Quellen: Bericht des Durban Monitoring the AIDS Pandemic (MAP) Network Symposium, The Status and Trends of the HIV/AIDS in the World vom Juli 2000 (Durban MAP Report; zur Situation in Afrika vgl. S. 3-5); International AIDS Vaccine Initiative (http://www.iavi.org); Deutsche Aids-Stiftung (http://www.aidsstiftung.de).

$\mathrm{Zu}$ den Ursachen von Aids in Südafrika vgl. die auch umfangreiche Reportage in der Süddeutschen Zeitung, München, vom 13./14.6.2001, S. 3.

8 Vgl. den Bericht in Guardian Unlimited vom 5.3.2001 unter http://www.guardianunlimited.co.uk/ international/story/0,3604,446465,00.html.

Zum Wortlaut seiner Rede zur Eröffnung der 13. Welt-Aids-Konferenz vgl. die Dokumentation in der Frankfurter Rundschau vom 28. Juli 2000, S. 9.

10

Zur Aids-Politik der Regierung Mbeki vgl. die Informationen des Department of Health unter http://www.doh.gov.za/aids/index.html. 
Nun ist die Immunschwäche nach wie vor unheilbar, doch seit Mitte der neunziger Jahre gibt es Präparatsmischungen, die das Leiden der Betroffenen erheblich lindern und ihr Leben verlängern. ${ }^{11}$ Bei einem regulären Verkaufspreis von mehr als US \$ 10.000 pro Jahr und Patient können sich die meisten Menschen den notwendigen Medikamenten-Cocktail der Patentinhaber in Afrika allerdings nicht leisten. Selbst preiswerte Nachahmungspräparate, die in Ländern wie Brasilien oder Indien zu einem Preis von unter US \$ 500 pro Jahr und Patient angeboten werden, übersteigen in der Regel das private Leistungsvermögen und sind auch aus öffentlichen Mitteln in den notwendigen Mengen kaum finanzierbar. ${ }^{12}$ Die südafrikanische Gesellschaft befindet sich damit nach vielen Jahrzehnten des politischen Ausnahmezustandes in einer akuten medizinischen Notlage, die durch die sozialen und wirtschaftlichen Folgeprobleme der Epidemie $^{13}$ noch zusätzlich verschärft wird. Erklärt diese Situation den Versuch der südafrikanischen Regierung, die Versorgung der Bevölkerung mit preiswerten Pharmazeutika per Gesetz sicherzustellen, geriet sie mit dem Amendment Act jedoch unversehens in einen Konflikt mit Inhabern bestehender pharmazeutischer Patente, die mit ihrer im Februar 1998 erhobenen Klage insbesondere eine Verletzung von Eigentumsrechten ${ }^{14}$ und der Freiheit der Berufsausübung ${ }^{15}$ reklamierten. Auf diplomatischer Ebene nahmen die USA, die Europäische Union und einzelne europäische Länder sogar schon im Vorfeld des eigentlichen Gesetzgebungsverfahrens Einfluss auf die Regierung des damaligen Staatspräsidenten Nelson Mandela, um die geplanten Änderungen des Arzneimittelrechts zu verhindern. Schließlich wurde die umstrittene Section 15C Medicines Act im April 1998 auch gegenüber der Welthandelsorganisation (WTO) vom Beauftragten des US-Präsidenten (U.S. Trade Representative, USTR) ${ }^{16}$ im Rahmen einer

Die Zahl der Todesfälle ist durch den Einsatz dieser Medikamente in einigen Ländern um bis zu $70 \%$ gesenkt worden.

12

In Brasilien, wo der kostenlose Zugang zu Aids-Medikamenten 1996 durch einen präsidialen Erlass gewährleistet wurde und staatliche Unternehmen etwa $30 \%$ der notwendigen Präparate in Form von Generika (Nachahmungspräparaten) herstellen, belaufen sich die Kosten des Gesundheitsministeriums für die 75.000 Patienten des Anti-Aids-Programms auf US \$ 400 Millionen; vgl. Durban MAP Report, a.a.O. (Fn. 6), S. 7/8. Zur mangelnden Finanzierbarkeit ähnlicher Programme in Afrika vgl. beispielsweise den Bericht der International Herald Tribune, New York/Washington, D.C., vom 6.4.2001, S. 5.

So unter anderem ein Anstieg in der Zahl von Waisenkindern, zunehmende Kinderarbeit, sinkende Lebenserwartung und sinkende Nahrungsmittelproduktion; vgl. hierzu etwa Durban MAP Report, a.a.O. (Fn. 6), S. 12-14; Süddeutsche Zeitung vom 13./14.6.2001, S. 3; Frankfurter Rundschau vom 20.7.2001, S. 30).

Section 25 Constitution of the Republic of South Africa, 1996, Act 108 of 1996 (Constitution Act).

Section 22 Constitution Act.

16

Der U.S. Trade Representative ist Kabinettsmitglied und ständiger Berater des US-Präsidenten in Außenhandelsfragen, vgl. Section 1 (b) (1) Government Organization and Employees Appendix 1, Reorganization Plan No. 3 of 1979, 5 U.S.C.A. App. 1 Reorg. Plan 3 1979. Seine Behörde unter- 
Anfrage zu Handelshemmnissen im südlichen Afrika angesprochen. Hintergrund dieser Initiative ist das Übereinkommen über handelsbezogene Aspekte der Rechte des geistigen Eigentums (Agreement on Trade-Related Aspects of Intellectual Property Rights - TRIPSÜbereinkommen), das aus der Uruguay-Runde der WTO hervorgegangen ist und weltweite Mindeststandards zum patentrechtlichen Schutz auch pharmazeutischer Produkte und Herstellungsverfahren anstrebt. ${ }^{17}$ Zwischen Mai 1998 und Dezember 1999 stand die Republik Südafrika überdies auf der Special 301 Watch List des USTR - einer Liste von Ländern, die in dem Verdacht stehen, mit ihrer Wirtschaftspolitik gegen internationale Abkommen oder bilaterale Verträge zu verstoßen und damit Handelsinteressen der Vereinigten Staaten im Bereich geistiger Eigentumsrechte $\mathrm{zu}$ verletzen. ${ }^{18}$ Diese Maßnahme wurde insbesondere mit der angestrebten Novellierung des Medicines Act begründet ${ }^{19}$ und erst im Vorfeld einer vorsichtigen Kursänderung in der US-amerikanischen Wirtschaftspolitik gegenüber den von Aids besonders stark betroffenen afrikanischen Ländern südlich der Sahara aufgehoben. ${ }^{20}$ Die Beachtung des TRIPS-Übereinkommens wird von den USA jedoch weiterhin eingefordert. ${ }^{21}$ Der starke Widerstand gegen die südafrikanische Gesetzesinitiative belegt, dass es um mehr als eine nationale Regelung des Arzneimittelrechts geht; nicht nur die gesundheitliche Versorgung von Menschen, sondern erhebliche wirtschaftliche Interessen sind von dem Amendment Act betroffen.

Obwohl nun der Konflikt zwischen den Inhabern pharmazeutischer Patente und der südafrikanischen Regierung mit der Klagerücknahme vorläufig beigelegt ist, verdient das Verfahren 4183/98 des High Court of South Africa (Transvaal Provincial Division) auch nach seinem Abschluss aus zwei Gründen eine genauere Untersuchung: (1) Der Ausgang

hält ein Büro bei der WTO (zu Einzelheiten über USTR vgl. http://www.ustr.gov/aboutustr/index.html).

Das Übereinkommen zur Errichtung der Welthandelsorganisation und die Schlussakte über die Ergebnisse der multilateralen Handelsverhandlungen der Uruguay-Runde (einschließlich des TRIPS-Übereinkommens, Anhang 1 C) wurden am 15.4.1994 in Marrakesch unterzeichnet und auch in Deutschland mit Gesetz vom 30.8.1994 (BGB1. II S. 1438 ff.) ratifiziert. Das Abkommen ist hier seit dem 1.1.1995 in Kraft (BGB1 II S. 456). Zum TRIPS-Übereinkommen vgl. Hubmann / Götting, Gewerblicher Rechtsschutz, 6. Aufl., 1998, S. 104 f.

Grundlage der Special 301 Watch List ist Section 182 Trade Act of 1974. Vgl. zum Special 301Verfahren auch USTR, Monitoring and Enforcing Trade Laws and Agreements (Fact Sheet vom 1.5.2000), S. 7 (zu finden unter http://www.ustr.gov) und Jakob, Die Zukunft US-amerikanischer unilateraler Section 301-Maßnahmen, GRUR Int. 2000, $715 \mathrm{ff}$.

Vgl. den Hinweis in USTR, 1999 National Trade Estimate Report on Foreign Trade Barriers (NTE), S. 382/383 (zu finden unter http://www.ustr.gov/pdf/nte-1999.pdf).

Vgl. Section 1 (a) der Executive Order No. 13155 des USTR vom 10.5.2000 (Access to HIV/AIDS Pharmaceuticals and Medical Technologies) der Clinton-Administration.

Im 2000 NTE (S. 371) wird das Ende der besonderen Beobachtung durch den USTR mit einer Verständigung zwischen beiden Regierungen bezüglich des Schutzes pharmazeutischer Patente begründet (zu finden unter http://www.ustr.gov/pdf/nte-2000.pdf). 
des Prozesses hat über die Grenzen Südafrikas hinaus erheblichen Einfluss auf den praktischen Umgang mit pharmazeutischen Patenten ausgeübt, ${ }^{22}$ ohne die durch die Klage aufgeworfenen rechtlichen Fragestellungen zu beantworten. Dies gilt sowohl für die innerstaatliche verfassungsrechtliche Zulässigkeit des Eingriffs in den patentrechtlichen Schutz pharmazeutischer Produkte und Herstellungsverfahren als auch die Zulässigkeit entsprechender Regelungen nach den Bestimmungen des internationalen TRIPS-Übereinkommens. Während sich die WTO mit der handelsrechtlichen Problematik in ähnlich gelagerten Fällen bereits auseinandersetzen muss, ${ }^{23}$ ist auch die Wahrscheinlichkeit vergleichbarer verfassungsrechtlicher Konflikte in anderen Ländern - möglicherweise sogar eine Neuauflage des Prozesses in Südafrika selbst - relativ groß. (2) Das Verfahren in Pretoria hat nicht nur den weltweiten Bemühungen um die Bekämpfung von Aids neue Impulse verliehen, ${ }^{24}$ sondern auch die Diskussion um die grundsätzliche Vertretbarkeit patentrechtlicher Schutzvorschriften im Bereich der pharmazeutischen Industrie und der Gentechnologie befruchtet. Für beide Punkte soll der vorliegende Beitrag einige Anregungen liefern. Hierzu werden das Spannungsverhältnis zwischen der Schutzpflicht eines Staates, die medizinische Versorgung seiner Bürger sicherzustellen, sowie den grundrechtlichen Ansprüchen der Menschen auf eine solche Versorgung einerseits und dem patentrechtlichen Schutz pharmazeutischer Produkte und Herstellungsverfahren andererseits beleuchtet (unten II.), einige Hintergründe und der Ablauf des Verfahrens vor dem High Court in Pretoria dargestellt (unten III.), die Erfolgsaussichten ähnlicher Klagen nach der verfassungsrechtlichen Lage in Südafrika bewertet (unten IV.) und die patentrechtlichen Ausnahmen des Medicines Act

So haben Pharmakonzerne wie GlaxoSmithKline, Merck, Boehringer-Ingelheim und Bristol Squibb-Meyer unter dem Eindruck des Verfahrens in Pretoria in Uganda, Gabun, Ruanda, Kamerun, Mali, Burundi, der Elfenbeinküste und im Senegal erhebliche Preisnachlässe auf Aidsmedikamente gewährt und sich gegenüber den Vereinten Nationen verpflichtet, diese Nachlässe auch weiteren armen Ländern einzuräumen. Kenia plant ein Gesetz, das sich eng an den umstrittenen Bestimmungen des südafrikanischen Medicines Act orientieren soll; vgl. Philadelphia Inquirer, Philadelphia, vom 18.4.2001 (http://inq.philly.com/content/inquirer/2001/04/18/national/ DRUGS18.html); Frankfurter Rundschau vom 18.4.2001, S. 1, 12.5.2001, S. 32, und 22.6.2001, S. 1 .

So etwa in Bezug auf den Umgang mit pharmazeutischen Patenten in Brasilien (patentrechtlicher Schutz von Produkten und Herstellungsverfahren nur bei lokaler Herstellung, nicht bei Import; vgl. die Auseinandersetzung zwischen den USA und Brasilien bei der WTO, WT/DS199/1 WT/DS199/4) und Indien (kein patentrechtlicher Schutz der Produkte sondern nur der Herstellungsverfahren).

Für den Kampf gegen die Aids-Epidemie in Afrika ist der kanadische Diplomat Stephen Lewis zum Sondergesandten der Vereinten Nationen ernannt worden. Er soll die weltweiten Bemühungen zur Eindämmung der Aids-Epidemie koordinieren; vgl. Frankfurter Rundschau vom 5.6.2001, S. 30. Außerdem soll ein internationaler „Anti-Aids-Fonds“ nach Plänen von UN-Generalsekretär Kofi Annan Entwicklungs- und Schwellenländern für Präventions- und Behandlungsmaßnahmen jährlich bis zu zehn Milliarden US \$ zur Verfügung stellen; vgl. Frankfurter Rundschau vom 22.6.2001, S. 1 . 
anhand der allgemeinen Standards und Ausnahmeregelungen des TRIPS-Übereinkommens überprüft (unten V.).

\section{Das Spannungsverhältnis zwischen medizinischer Versorgung und Patentschutz}

Ob Wunderheiler oder High-Tech-Medizin: Leben und Gesundheit des Menschen sind in hohem Maße auf ärztliche Hilfe und die Versorgung mit Arzneimitteln angewiesen. Als Rechtsgüter genießen Leben und Gesundheit in den meisten Gesellschaften dabei zivilrechtlichen, strafrechtlichen und verfassungsrechtlichen Schutz; ${ }^{25}$ ihr überragender Stellenwert wird aber auch erkennbar an der Bedeutung, die etwa den medizinischen Systemen (von der Ausbildung und Zulassung von Ärzten bis hin zum Bau von Krankenhäusern), der medizinischen Forschung, der Regulierung des Arzneimittelverkehrs, dem Aufbau und der Aufrechterhaltung von Kranken- und Unfallversicherungssystemen sowie der Überwachung von Lebens- und Genussmitteln beigemessen wird. ${ }^{26}$ Dabei tritt beispielsweise in Deutschland in Bezug auf Leben und körperliche Unversehrtheit neben das grundrechtliche Abwehrrecht des Einzelnen gegenüber staatlichen Eingriffen (status negativus) eine positive Schutzpflicht des Staates, ${ }^{27}$ das Leben und die Gesundheit seiner Bürger sicherzustellen. Dies ergibt sich auch aus dem Sozialauftrag des Grundgesetzes ${ }^{28}$ und statuiert im Zusammenhang mit der Garantie der Menschenwürde (Art. 1 Abs. 1 GG) einen verfassungsrechtlich gewährleisteten Mindeststandard menschlicher Existenz. ${ }^{29}$ Ob Art. 2 Abs. 2 S. 1 GG dabei subjektive Leistungsansprüche auf medizinische Versorgung begründet oder nur ein ,wertsetzendes Programm für staatliches Handeln“ ${ }^{30}$ liefert, ist dabei allerdings noch ungeklärt.

Wirtschaftliche Interessen sind die zweite Komponente des Konflikts. Im Brennpunkt der Bemühungen um Leben und Gesundheit steht heute neben der eigentlichen medizinischen Dienstleistung die Entwicklung, Produktion und Bereitstellung medizinischer Technik und

25

26

27

28

29

30

Vgl. etwa in Deutschland $§ 823$ Abs. 1 BGB; $§ \S 211$ ff. StGB; Art. 2 Abs. 2 S. 1 GG.

Vgl. etwa in Deutschland Art. 74 Nr. 19, 19a und 20 GG.

Kunig, Rn. 54 f. zu Art. 2, in: von Münch / Kunig, GGK I, 5 Aufl., 2000

Art. 20 Abs. 1, 28 Abs. 1, 79 Abs. 3 GG.

Dürig in: Maunz-Dürig, Komm. z. GG (Stand: März 2001), Art. 2 Rn. 26 f.; Kannengießer, in: Schmidt-Bleibtreu / Klein, Kommentar zum Grundgesetz, 9. Aufl., 1999, Art. 2 Rn. 20b; Jarass, in: Jarass / Pieroth, Grundgesetz für die Bundesrepublik Deutschland, 5. Aufl., 2000, Art. 2 Rn. 60; BVerwGE 1, 59, 162.

So etwa Kunig, Rn. 60 zu Art. 2, in: von Münch / Kunig, GGK I, 5. Aufl., 2000. Das Bundesverfassungsgericht sieht einen Anspruch auf sachgerechte Ausgestaltung staatlicher Krankeneinrichtungen (BVerfGE 57, 70, 99). 
pharmazeutischer Hilfsmittel. Hier hat die moderne Medizin in den vergangenen Jahrzehnten große Fortschritte erzielt und sich zugleich zu einem eigenständigen Industriesektor mit Milliardenumsätzen entwickelt: Heilmittel sind inzwischen zu einer Handelsware geworden, die (ungeachtet ihrer besonderen Bedeutung für den Menschen) nach betriebswirtschaftlichen Gesichtspunkten hergestellt und vermarktet wird. Ein wichtiges Kriterium im weltweiten Wettbewerb der Pharmakonzerne ist dabei das Wissen um die Zusammensetzung der verschiedenen Wirkstoffe und der für die Produktion erforderlichen Herstellungsverfahren. Das hohe wirtschaftliche Interesse an der Verwertung dieser Informationen ist patentrechtlich geschützt; Staaten erteilen Patentinhabern dabei die ausschließliche, aber zugleich auch zeitlich ${ }^{31}$ und territorial ${ }^{32}$ begrenzte Befugnis, bestimmte pharmazeutische Erfindungen zu benutzen. ${ }^{33}$ Verfassungsrechtlich unterliegt das Recht des Erfinders in vielen Rechtsordnungen der Eigentumsgarantie (sog. Theorie vom geistigen Eigentum), ${ }^{34}$ und auch in Deutschland gewährt Art. 14 Abs. 1 GG dem Rechtsinhaber jedenfalls vermögensrechtlichen Schutz. ${ }^{35}$ Werden Patentinhaber durch rechtlich geschützte Verfahren, Produkte, Vertriebswege und Märkte dabei in die Lage versetzt, möglichst hohe Verkaufserlöse zu erzielen, liefern ausschließliche Nutzungsrechte aber zugleich auch einen wirtschaftlichen Anreiz, Mittel für die Erforschung von Krankheiten und die Entwicklung neuer Medikamente und Behandlungsmethoden zur Verfügung zu stellen. ${ }^{36}$ Die International Federation of Pharmaceutical Manufacturers (IFPMA) geht davon aus, dass sogar bis zu $65 \%$ der bislang entwickelten pharmazeutischen Produkte ohne Patentschutz nicht auf den Markt gekommen wären. ${ }^{37}$ Das Patentrecht dient damit sowohl dem wirtschaftli-

Die Schutzdauer beträgt in der Regel 20 Jahre, vgl. z. B. § 16 Patentgesetz (PatG) und Art. 33 TRIPS-Übereinkommen.

Zum Territorialprinzip im Patentrecht vgl. Ullrich, Technologieschutz nach TRIPS: Prinzipien und Probleme, GRUR Int. 1995, 623, 624 ff.

Patentfähig sind sowohl pharmazeutische Herstellungs- und Anwendungsverfahren (als sogenannte Verfahrenspatente) als auch die Arzneimittel selbst (als sogenannte Sachpatente), § 5 Abs. 2 S. 2, § 3 Abs. 3 PatG; vgl. Hubmann / Götting, a.a.O. (Fn. 17), S. 116.

Zur rechtlichen Lage in Frankreich und den USA vgl. Hubmann / Götting, a.a.O. (Fn. 17), S. 20 ff.

Bryde, Rn. 17 zu Art. 14, in: von Münch / Kunig, GGK I, 5 Aufl., 2000; Papier, in: Maunz-Dürig, Komm. zum GG (Stand: März 2001), Art. 14 Rn. 196; BVerfGE 36, 281 (290 ff.); SchmidtBleibtreu, in: Schmidt-Bleibtreu / Klein, Kommentar zum Grundgesetz, 9. Aufl., 1999, Art. 14 Rn. 3e.

$\mathrm{Zu}$ dem Zusammenhang zwischen Forschung und Patentschutz vgl. Heath, Bedeutet TRIPS wirklich eine Schlechterstellung von Entwicklungsländern?, GRUR Int. 1996, 1169, 1171 f., 1175.

Directorate General of Drug and Food Control / WHO, The Trips Agreement and Pharmaceuticals, Report of an ASEAN Workshop on the TRIPS Agreement and its Impact on Pharmaceuticals (Jakarta, 2.-4.5.2000), S. 13 - Tabelle 3 - nach einer Studie von Mansfield, Management Science, 1986. 
chen Interesse der Patentinhaber als auch (mittelbar) dem Interesse der Allgemeinheit an gewinnfinanzierter Forschung und Innovation im privaten Sektor. ${ }^{38}$

Tatsächlich investieren die Pharmaunternehmen erhebliche Summen in diesen Bereich ${ }^{39}$ und verweisen mit Blick auf das Verfahren in Pretoria gerne auf den Zusammenhang zwischen Forschung und dem Schutz geistigen Eigentums. Die Formel „ohne Patenschutz keine Forschung “ ${ }^{40}$ erscheint indes überzogen. Auch ist die geschilderte positive Wirkung ausschließlicher Nutzungsrechte auf die pharmazeutische Forschung nicht unumstritten. So argumentieren Kritiker patentrechtlicher Schutzvorschriften etwa unter Hinweis auf die in den letzten drei Jahrzehnten von Südostasien angetriebene Entwicklung im Bereich der Mikrotechnologie, dass Innovation häufig von Imitation und anschließender Verbesserung angetrieben werde; langjährige Patentrechte erdrosseln hiernach diese durch den freien Wettbewerb von Ideen erzeugte Dynamik. ${ }^{41}$ Dies benachteilige insbesondere die Entwicklungsländer (infant industry-Argument). ${ }^{42}$ Außerdem wird darauf hingewiesen, dass ein großer Teil der Kosten für die Entwicklung lebenswichtiger Medikamente von staatlichen Forschungsbudgets getragen werde und damit steuerfinanziert sei. Dies treffe insbesondere auf die sogenannten antiretroviralen Aids-Medikamente ${ }^{43} \mathrm{zu}$, die teilweise im Zusammenhang mit der Erforschung anderer Krankheiten im Rahmen von öffentlich geförderten Projekten in den USA entwickelt worden sind. ${ }^{44}$ Kritiker von Pharmapatenten machen auch

Directorate General of Drug and Food Control / WHO, The Trips Agreement and Pharmaceuticals, Report of an ASEAN Workshop on the TRIPS Agreement and its Impact on Pharmaceuticals (Jakarta, 2.-4.5.2000), S. 10, 57; Liebig, Der gierige Griff nach dem geistigen Eigentum, in: Frankfurter Rundschau vom 11.5.2001, S. 9; Thums, Patentschutz für Heilverfahren?, GRUR Int. 1995, 277, 278, 284.

Straus, Bedeutung des TRIPS für das Patentrecht, GRUR Int. 1996, 179, 180.

So Cornelia Yzer, Hauptgeschäftsführerin des Verbandes Forschender Arzneimittelhersteller, vgl. den Bericht in der Frankfurter Rundschau vom 20.4.2001, S. 1.

Directorate General of Drug and Food Control / WHO, The Trips Agreement and Pharmaceuticals, Report of an ASEAN Workshop on the TRIPS Agreement and its Impact on Pharmaceuticals (Jakarta, 2.-4.5.2000), S. 7/8; Mansfield, Unauthorized Use of Intellectual Property: Effects on Investment, Technology, Transfer and Innovation, in: Wallerstein / Mogee / Schoen (Hrsg.), Global Dimensions of Intellectual Property Rights in Science and Technology, 1993, 107, 133 ff.

Rahn, Die Bedeutung des gewerblichen Rechtsschutzes für die wirtschaftliche Entwicklung: die japanischen Erfahrungen, GRUR Int. 1982, 581 ff.; Pacón, Was bringt TRIPS den Entwicklungsländern?, GRUR Int. 1995, 875.

Medikamente, die unmittelbar auf die Aids-Infektion abzielen.

So etwa das Medikament Zerit, das von William Prusoff (Yale) entwickelt und patentiert worden ist und von Bristol-Myers Squibb gegen Patentgebühren vermarktet wird. Nach Protesten von Studenten in Yale hat die Firma den Preis des Medikaments von US \$ 1,50/Tablette auf US \$ 0,15/Tablette gesenkt; vgl. den Bericht in Philadelphia Inquirer vom 18.4.2001 unter http://inq. philly.com/content/inquirer/2001/04/18/national/DRUGS18.html. Zum Argument der Entwicklungskosten vgl. auch International Council of AIDS Service Organizations (ICASO), Compul- 
geltend, dass die Entwicklungskosten vieler Präparate von den Herstellern seit ihrer Markteinführung schon längst wieder „,eingefahren“ wurden; ${ }^{45}$ weiterer patentgeschützter Gewinn (der Verkaufspreis dieser Medikamente übersteigt die Herstellungskosten teilweise um mehr als das Zehnfache) sei also Profit auf Kosten von Kranken. Schließlich werden die Forschungsbemühungen der Privatwirtschaft mit einem Vergleich zu den Werbeetats der Pharmakonzerne relativiert; letztere übersteigen die Budgets für research and development (R\&D) zum Teil um das Doppelte. Insgesamt fällt der Konflikt um pharmazeutische Patente überdies in eine Phase, in der Patentrechte und die aus ihnen resultierenden starken Marktpositionen einzelner Unternehmen in der öffentlichen Meinung sehr kontrovers diskutiert werden. ${ }^{46}$

Betrachtet man nun die hier kurz angedeutete gegenläufige Interessenlage unter dem Blickwinkel westlicher Industriegesellschaften, so ist einzuräumen, dass sich das Spannungsverhältnis zwischen medizinischer Versorgung und Patentschutz bislang nur andeutet. In reichen Gesellschaften sind die hohen Gewinne der Pharmaunternehmen tolerabel, weil sozialstaatliche Sicherungssysteme die Versorgung der Menschen (noch) gewährleisten können. ${ }^{47}$ Die Aids-Katastrophe in Südafrika zeigt jedoch, dass sich die Schere zwischen der wissenschaftlich möglichen und der gesellschaftlich finanzierbaren medizinischen Versorgung in weniger entwickelten Gesellschaften bereits unerträglich weit geöffnet hat. Dabei sind im Rahmen der hier erforderlichen Abwägung zwischen dem staatlichen Interesse an der Versorgung der Bevölkerung mit lebensnotwendigen Medikamenten und dem grundrechtlich geschützten Individualinteresse der von der Seuche Betroffenen an medizinischer Versorgung einerseits ${ }^{48}$ und dem (gegenläufigen) Interesse der Patentinhaber an einer möglichst ertragreichen Nutzung von pharmazeutischen Erfindungen und dem Interesse der Allgemeinheit an privatfinanzierter Forschung andererseits ${ }^{49}$ die besonderen Rahmenbedingungen in unterentwickelten Gesellschaften zu berücksichtigen. Dies zeigt sich besonders deutlich am Beispiel Aids: Afrika vertritt hier einen Marktanteil von nur etwa $1 \%$ am Verkauf von Aids-Medikamenten; die Gewinnaussichten der Patentinhaber

sory Licensing and Parallel Importing (Background Paper, July 1999), zu finden unter http://www. icaso.org/compulsory_english.html.

45

46

47

48

49

So etwa von AZT, ddI, d4T, Lamivudin und Fluconazol.

Ein Parallelfall ist die Kontroverse um den patentrechtlichen Schutz von Computersoftware in den USA (Microsoft).

Die Kontroversen um eine Reform des Gesundheitswesens und Beitragserhöhungen der Krankenkassen in Deutschland zeigen jedoch, dass die Kosten für pharmazeutische Produkte (nach Schätzungen der Apothekenverbände mehr als 40 Milliarden Mark in 2001 nur für die Gesetzlichen Krankenkassen) auch wohlhabende Gesellschaften erheblich belasten; vgl. den Bericht in der Frankfurter Rundschau vom 1.8.2001, S. 1.

Kriterien: möglichst geringer Verkaufspreis und hohe Zugänglichkeit von Medikamenten zur Behandlung von Aids-Patienten.

Kriterium: hohe Gewinne durch die ausschließliche Verwertung von Wissen. 
sind damit äußerst gering, obwohl schätzungsweise $71 \%$ der weltweit von der Epidemie betroffenen Menschen südlich der Sahara leben. ${ }^{50}$ Gleichzeitig sind die Investitionen der Pharmaunternehmen in die lokale Forschung praktisch zu vernachlässigen. ${ }^{51}$ Die beiden wesentlichen Anreize zur Errichtung und Beachtung eines strengen Patentregimes - die Herausbildung eines nationalen Forschungspotentials und/oder die Teilhabe an fremden Forschungsleistungen - entfallen damit in aller Regel.

Eine weitere Besonderheit in der Auseinandersetzung um pharmazeutische Patente ist schließlich die internationale Dimension des Konflikts. Während medizinische Versorgung ihrer Natur nach eigentlich eine nationale Aufgabe ist und - wie auch die Versorgung mit Nahrungsmitteln oder Wasser - grundsätzlich auf dieser Ebene gelöst werden muss, können sich patentrechtliche Niveauunterschiede in einzelnen Regionen mittelbar auf den Weltmarkt auswirken. So war die Befürchtung, billige Medikamente aus Ländern mit einem herabgesetzten Patentschutz könnten über schwarze Märkte den Handel in anderen Regionen beeinträchtigen, sicherlich ein wichtiges Motiv der Klage vor dem High Court in Pretoria sowie des internationalen politischen Widerstandes gegen den südafrikanischen Amendment Act. In Südafrika selbst gab es die Sorge, dass sich die Änderungen des Patentrechts im pharmazeutischen Bereich insgesamt negativ auf die Bereitschaft ausländischer Unternehmen auswirken könnten, in die Wirtschaft des Landes zu investieren. ${ }^{52}$ Auf rechtlicher Ebene schafft das Ausscheren einzelner Rechtsordnungen zudem Präzedenzfälle, die die im Rahmen der WTO mit dem TRIPS-Übereinkommen angestrebte weltweite Homogenität patentrechtlicher Schutzvorschriften gefährdet.

\section{Case No. 4183/98 des High Court of South Africa}

Der umstrittene Medicines and Related Substances Control Amendment Act wurde von beiden Kammern des südafrikanischen Parlaments im Oktober und November 1997 verabschiedet und am 25.11.1997 vom damaligen Staatspräsidenten Nelson Mandela unterzeich-

Vgl. die Informationen der Deutschen Aids-Stiftung unter http://www.aids-stiftung.de. Dabei ist zu berücksichtigen, dass die für den Vertrieb von Arzneimitteln notwendige Infrastruktur in vielen Ländern sehr schlecht ist (was zu Versorgungsengpässen führt). Außerdem fehlen häufig die für die Bekämpfung von Aids dringend erforderlichen Kliniken und ambulanten Behandlungsmöglichkeiten. Die Versorgung mit notwendigen Arzneimitteln leidet aber auch unter hausgemachten bürokratischen Hemmnissen, etwa langwierigen Zulassungsverfahren.

Directorate General of Drug and Food Control / WHO, The Trips Agreement and Pharmaceuticals, Report of an ASEAN Workshop on the TRIPS Agreement and its Impact on Pharmaceuticals (Jakarta, 2.-4.5.2000), S. 11. 18.4.2001 unter http://www.guardian.co.uk/aids/story/0,7369,474484,00.html sowie unter http:// www.guardian.co.uk/aids/story/0,7369,474361,00.html. 
net. ${ }^{53}$ Im Wesentlichen bewirkt die Novelle vier wichtige Änderungen des bisherigen südafrikanischen Arzneimittelrechts: (1) Die zur Abgabe von Medikamenten berechtigten Personen unterliegen gegenüber ihren Kunden einer Informationspflicht bezüglich des Angebots an sogenannten „Generika“ (in der Regel preisgünstige Nachahmungspräparate von patentierten Markenarzneimitteln), soweit die Schutzfrist der Originale nach den Bestimmungen des Patents Act inzwischen abgelaufen ist (off-patent products) oder Nachahmungspräparate während der Laufzeit von Patenten aufgrund von Sondergenehmigungen am Markt zugelassen sind. ${ }^{54}$ Sie sind darüber hinaus verpflichtet, vorrangig Generika abzugeben, wenn nicht (a) der Kunde ausdrücklich das Produkt des Erstherstellers verlangt; ${ }^{55}$ (b) der behandelnde Arzt auf dem Rezept Generika ausdrücklich ausschließt; ${ }^{56}$ (c) das Generikum teurer ist als das Markenarzneimittel; ${ }^{57}$ oder (d) der Medicines Control Council - eine Aufsichtsbehörde zur Überwachung des Arzneimittelmarktes - erklärt hat, dass ein bestimmtes Medikament in Südafrika auch nach Ablauf einer patentrechtlichen Schutzfrist nicht durch Generika ersetzt werden darf. ${ }^{58}$ (2) Ein Pricing Committee, vom Gesundheitsminister eingerichtet, ${ }^{59}$ wird künftig die Verkaufspreise aller am südafrikanischen Markt angebotenen Medikamente überprüfen und gegebenenfalls Empfehlungen zur Einführung von Preiskontrollen (single exit prices) aussprechen. Es entwickelt darüber hinaus Vorschläge zur Einführung einheitlicher Gebühren, die bei Abgabe von Medikamenten erhoben werden dürfen (dispensing fees). Der Gesundheitsminister kann diese Empfehlungen im Verordnungswege umsetzen. ${ }^{60}$ (3) Der Gesundheitsminister wird er-

Republic of South Africa, Government Gazette No. 18505 vom 12.12.1997 (zu finden unter http:// www.gov.za/acts/1997/a90-97.pdf.

Section 22F (1) Medicines Act: „Subject to subsections (2), (3) and (4), a pharmacist shall - (a) inform all members of the public who visit his or her pharmacy with a prescription for dispensing, of the benefits of the substitution for a branded medicine of an interchangeable multi-source medicine; and (b) ...".

Section 22F (1) Medicines Act: „Subject to subsections (2), (3) and (4), a pharmacist shall - ... (b) dispense an interchangeable multi-source medicine instead of the medicine prescribed by a medical practitioner, dentist, practitioner, nurse or other person registered under the Health Professions Act, 1974, unless expressly forbidden by the patient to do so“.

Section 22F (4) Medicines Act: „A pharmacist shall not sell an interchangeable multi-source medicine - (a) if the person prescribing the medicine has written in his or her own hand on the prescription the words 'no substitution' next to the item prescribed; ...".

Section 22F (4) Medicines Act: „A pharmacist shall not sell an interchangeable multi-source medicine - (b) if the retail price of the interchangeable multi-source medicine is higher than that of the prescribed medicine; or ...".

Section 22F (4) Medicines Act: „A pharmacist shall not sell an interchangeable multi-source medicine - (c) where the product has been declared not substitutable by the council“".

Section 22G (2) and (3) Medicines Act: ,(2) The Minister may, on the recommendation of the pricing committee, make regulations - (a) on the introduction of a transparent pricing system for all medicines and Scheduled substances sold in the Republic; (b) on an appropriate dispensing fee 
mächtigt, die Einfuhr und Produktion von Generika (compulsory licensing) sowie die Einfuhr von Markenarzneimitteln zu Preisen unterhalb des südafrikanischen Preisniveaus (parallel importation) entgegen den Bestimmungen des Patentgesetzes durch Verordnung zu regeln, um die medizinische Versorgung der Bevölkerung sicherzustellen. ${ }^{61}$ Beide Maßnahmen betreffen Markenarzneimittel während der Laufzeit von Patenten (on-patent products). (4) Medikamente, die im staatlichen Gesundheitswesen gebraucht werden, dürfen zu möglichst günstigen Konditionen am Weltmarkt eingekauft werden (international tendering for medicines). ${ }^{62}$

Die PMA und 39 in Südafrika ansässige pharmazeutische Unternehmen erhoben im Februar 1998 beim High Court of South Africa ${ }^{63}$ in Pretoria Klage gegen die südafrikanische Regierung, beide Kammern des Parlaments, die Provinz Gauteng, den Medicines Control Council sowie das nationale Patentamt und erwirkten zunächst eine einstweilige Verfügung gegen das Inkrafttreten des Amendment Act. In der Sache konzentrierte sich die Klage dabei im Wesentlichen auf die eben geschilderten Bestimmungen des Gesetzes. Die Kläger machten geltend, dass die in Section 15C Medicines Act verankerte Verordnungsbefugnis entgegen den verfassungsrechtlichen Vorgaben ${ }^{64} \mathrm{zu}$ unbestimmt sei (lack of due process): Der Gesundheitsminister könne die Rahmenbedingungen, die eine Versorgung mit

to be charged by a pharmacist or by a person licensed in terms of section $22 \mathrm{C}$ (1) (a). (3) (a) The transparent pricing system contemplated in subsection (2) (a) shall include a single exit price which shall be published as prescribed, and such price shall be the only price at which manufacturers shall sell medicines and Scheduled substances to any other person other than the State. (b) No pharmacist or person licensed in terms of section $22 \mathrm{C}$ (1) (a) shall sell a medicine at a price greater than the price contemplated in paragraph (a). (c) Paragraph (b) shall not be construed as preventing a pharmacist or person licensed in terms of this Act to charge a dispensing fee as contemplated in subsection (2) (b)“.

Section 15C Medicines Act: „The minister may prescribe conditions for the supply of more affordable medicines in certain circumstances so as to protect the health of the public, and in particular may - (a) notwithstanding anything to the contrary contained in the Patents Act, 1978 (Act No. 57 of 1978), determine that the rights with regard to any medicine under a patent granted in the Republic shall not extend to acts in respect of such medicine which has been put onto the market by the owner of the medicine, or with his or her consent; (b) prescribe the conditions on which any medicine which is identical in composition, meets the same quality standard and is intended to have the same proprietary name as that of another medicine already registered in the Republic, but which is imported by a person other than the person who is the holder of the registration certificate of the medicine already registered and which originates from any site of manufacture of the original manufacturer as approved by the council in the prescribed manner, may be imported; (c) prescribe the registration procedure for, as well as the use of, the medicine referred to in paragraph (b)“.

Section 1 (4) Medicines Act: „International tendering for medicines shall be allowed in the prescribed manner and on the prescribed conditions".

In der deutschen Gerichtsorganisation ist der High Court etwa vergleichbar mit einem Landgericht.

64

Sections 43 und 44 Constitution Act. 
erschwinglichen Medikamenten (affordable medicines) in Südafrika sicherstellen sollen, entgegen den Bestimmungen des Patents Act festlegen, ohne dass die Ermächtigungsgrundlage dabei hinreichend konkrete Bindungen (,policy considerations or sufficient guidelines") aufweise. ${ }^{65}$ Die Verordnungsbefugnis verletze überdies die Eigentumsgarantie, ${ }^{66}$ da sie eine entschädigungslose Enteignung des an pharmazeutischen Patenten bestehenden geistigen Eigentums erlaube, ${ }^{67}$ und diskriminiere die Inhaber pharmazeutischer Patente entgegen den Bestimmungen des internationalen TRIPS-Übereinkommens, ${ }^{68}$ das auch vom südafrikanischen Gesetzgeber zu beachten sei. ${ }^{69} \mathrm{Zu}$ unbestimmt sei auch die mit Section 1 (4) Medicines Act eingeführte Verordnungsbefugnis bezüglich des staatlichen Einkaufs von Medikamenten auf dem internationalen Markt; ${ }^{70}$ sie benachteilige überdies nationale Patentinhaber und verletze daher das verfassungsrechtliche Gleichbehandlungsgebot. $^{71}$ Die mit Section 22F Medicines Act vorgeschriebene bevorzugte Abgabe von Medikamenten mit gleicher Wirkungsweise (generic substitution) sei ebenfalls zu unbestimmt, ${ }^{72}$ verletze das Gleichbehandlungsgebot ${ }^{73}$ und die Eigentumsgarantie $^{74}$ zu Lasten der Inhaber pharmazeutischer Patente und das Recht der freien Meinungsäußerung ${ }^{75}$ sowie die Freiheit der Berufsausübung ${ }^{76}$ des zur Abgabe von Medikamenten berechtigten Personenkreises. Als Verletzung des Gleichbehandlungsgebots und der Freiheit der Berufsausübung von Herstellern pharmazeutischer Produkte und abgabeberechtigten Personen wurden auch die aufgrund von Section 22G (2) und (3) Medicines Act möglichen Preiskontrollen und die Einführung einheitlicher Gebühren für die Abgabe von Medikamenten angegriffen. Schließlich wurde vorgetragen, dass der Gesetzgeber die Öffentlichkeit bei der

Ziff. 2.1 und 2.2 Notice of Motion (case number 4183/98). Vgl. auch Guardian Unlimited vom 5.3.2001 unter http://www.guardianunlimited.co.uk/international/story/0,3604,446465,00.html. Section 25 Constitution Act.

Ziff. 2.3 Notice of Motion (case number 4183/98).

Art. 27 TRIPS-Übereinkommen i.V.m. Sections 44 (4) und 231 (2), (3) Constitution Act. Die Republik Südafrika ist dem TRIPS-Übereinkommen mit Inkrafttreten des Intellectual Property Laws Amendment Act, No. 38 of 1997 beigetreten.

Ziff. 2.4 Notice of Motion (case number 4183/98).

Ziff. 3.1 Notice of Motion (case number 4183/98).

Ziff. 3.2 Notice of Motion (case number 4183/98) unter Hinweis auf Section 9 Constitution Act.

.

Ziff. 4.2 Notice of Motion (case number 4183/98).

Ziff. 4.3 Notice of Motion (case number 4183/98).

Ziff. 4.4 Notice of Motion (case number 4183/98). Section 16 (1) (b) Constitution Act erklärt: „Everyone has the right to freedom of expression, which includes - (b) freedom to receive or impart information or ideas". citizen has the right to choose their trade, occupation or profession freely. The practice of a trade, occupation or profession may be regulated by law". 
Beratung des Amendment Act nicht hinreichend beteiligt habe. ${ }^{77}$ Jenseits rechtlicher Bedenken führen die Zulassung von Generika und staatlich verordnete Preise nach Ansicht der am Weltmarkt etablierten Pharmaunternehmen zu Qualitätseinbußen und gefährden damit letztlich die Arzneimittelsicherheit. ${ }^{78}$

Die beklagte südafrikanische Regierung verwies demgegenüber auf ihre verfassungsrechtliche Pflicht, die bestehenden grundrechtlichen Ansprüche der Menschen auf medizinische Versorgung, Wasser und soziale Absicherung durch geeignete Maßnahmen sicherzustellen. ${ }^{79}$ Die angegriffenen Bestimmungen des Amendment Act seien Bestandteil einer umfassenden Initiative zur Senkung der Kosten im Gesundheitswesen und zwingend notwendig, da die finanziellen Ressourcen des Staates nicht ausreichten, um die beispielsweise durch Aids ausgelösten Probleme ohne Eingriffe in den bestehenden südafrikanischen Arzneimittelmarkt zu bewältigen. Angebote der Pharmakonzerne, die Preise bestimmter patentgeschützter Präparate freiwillig zu senken, gingen zwar in die richtige Richtung, doch sei das Preisniveau angesichts der Zahl von Betroffenen nach wie vor viel zu hoch; ${ }^{80}$ außerdem dürfe sich das staatliche Gesundheitswesen nicht von Preisnachlässen abhängig machen, die von den Herstellern in Zukunft nach Belieben gewährt oder versagt werden könnten. Schließlich seien die beiden am meisten angegriffenen Instrumente - Zwangslizenzen und Parallelimporte - vom TRIPS-Übereinkommen jedenfalls in nationalen Notlagen gedeckt; ${ }^{81}$ parallel importation und staatliche Preiskontrollen würden sogar von westlichen

Ziff. 10.1 Notice of Motion (case number 4183/98) unter Hinweis auf Sections 59 (1) und 72 (1) Constitution Act.

Directorate General of Drug and Food Control / WHO, The Trips Agreement and Pharmaceuticals, Report of an ASEAN Workshop on the TRIPS Agreement and its Impact on Pharmaceuticals (Jakarta, 2.-4.5.2000), S. 13; so auch Heath, a.a.O. (Fn. 36), S. 1175.

Section 27 Constitution Act bestimmt: ,(1) Everyone has the right to have access to - (a) health services, including reproductive health care; (b) sufficient food and water; and (c) social security, including, if they are unable to support themselves and their dependants, appropriate social assistance. (2) The state must take reasonable legislative and other measures, within its available resources, to achieve the progressive realisation of each of these rights. (3) No one may be refused emergency medical treatment".

Vgl. die Stellungnahme von Ayanda Ntsaluba (Director General des südafrikanischen Gesundheitsministeriums) gegenüber Guardian Unlimited vom 5.3.2001 unter http://www.guardianunlimited.co.uk/international/story/0,3604,446465,00.html: „It doesn't help for them to say we will reduce the cost by $60 \%$ because it's still unaffordable for developing countries. The tender issue is a very strange argument. We have done a study that says that even if you reduce the cost of triple therapy in treating HIV by $80 \%$ the public sector could not afford it“".

Art. 6 des TRIPS-Übereinkommens - Erschöpfung - (parallel importation) und Art. 31 des TRIPS-Übereinkommens - sonstige Benutzung ohne Zustimmung des Rechtsinhabers - (compulsory lisencing). 
Industrienationen zur Senkung der Kosten im Gesundheitswesen eingesetzt. ${ }^{82}$ Obwohl die Regierung auch erkennen ließ, dass die Novelle in einzelnen Punkten vielleicht noch einmal überarbeitet werden müsste, gab sie sich sicher, spätestens bei einem erneuten Anlauf erfolgreich zu sein.

Hierzu kam es nicht. Der enorme öffentliche Druck, der von Interessenverbänden, internationalen Hilfsorganisationen und vielen ausländischen Regierungen nach den ersten Verhandlungstagen im März 2001 vor dem High Court auf die Hersteller pharmazeutischer Produkte ausgeübt wurde, ${ }^{83}$ bewirkte weltweit einen ungeahnten Imageverlust der Pharmaindustrie und schuf auch bei den in Pretoria klagenden Unternehmen die Bereitschaft, mit der südafrikanischen Regierung eine außergerichtliche Einigung zu erzielen. Zudem verschlechterte sich die prozessuale Situation der Kläger durch die Entscheidung des Gerichts, die Treatment Access Campaign (TAC), eine südafrikanische Aids-Selbsthilfeorganisation, als amicus curiae ${ }^{84}$ zum Verfahren zuzulassen. Unter Hinweis auf die Forschungstätigkeit von Universitäten und öffentlich geförderten Krebsforschungszentren in den USA widersprachen dabei TAC-Vertreter der Argumentation der Pharmaunternehmen, dass der patentrechtliche Schutz von Aids-Medikamenten erforderlich sei, um die Entwicklungskosten der fünf wichtigsten Präparate zu finanzieren. Der Staat könne die Ausgaben im Gesundheitssektor durch Parallelimporte ohne Verstoß gegen das TRIPS-Übereinkommen erheblich senken und die medizinische Versorgung der Bevölkerung damit spürbar verbessern. Auf verfassungsrechtlicher Ebene wiege das Recht der Betroffenen auf Leben ${ }^{85}$ und medizinische Versorgung ${ }^{86}$ sowie die Menschenwürde ${ }^{87}$ schwerer als die wirtschaftlichen Interessen der Patentinhaber. ${ }^{88}$ Das Gericht gab zu erkennen, dass es den Vortrag der TAC als von

Zur Position der südafrikanischen Regierung vgl. das Memorandum on the Objects of the Medicines and Related Substances Control Amendment Bill, 1997 (zu finden als Anhang zum Gesetzentwurf des Gesundheitsministeriums, S. 44/45 unter http://www.gov.za/bills/1997/b72-97/pdf) sowie eine Stellungnahme von Gesundheitsministerin Tschabalala-Msimang in Business Day, Johannesburg, zu finden unter http://www.bday.co.za/bday/content/direct/1,3523,832452-60960,00.html.

Die Klage ist international heftig kritisiert worden, so etwa von der Föderation der Rotkreuz- und Rothalbmondgesellschaften und der Hilfsorganisation „Ärzte ohne Grenzen“; vgl. den Bericht in der Frankfurter Rundschau vom 13.3.2001, S. 7. Auch die Bundesregierung hat die am Verfahren beteiligten deutschen Pharmakonzerne aufgefordert, die Klage zurückzunehmen und eine Einigung mit der südafrikanischen Regierung zu erzielen. Juristischer Berater des Gerichts.

Section 11 Constitution Act.

86

Section 27 Constitution Act.

87

Section 10 Constitution Act.

88

Vgl. etwa die Eidesstattlichen Versicherungen von James Packard Love (Centre for Study of Responsive Law, Washington, D.C.), Carmen Perez-Casas (Médecins Sans Frontières), Prof. Andrew Gray (University of Durban-Westville) und Theodora Steele (TAC). 
den Klägern zugestanden werten würde, wenn diese nicht die behaupteten Tatsachen im einzelnen widerlegten. ${ }^{89}$ Die mündliche Verhandlung wurde sodann auf den 18. April 2001 vertagt, um den Pharmaunternehmen Gelegenheit zu geben, sich auf diese neue Situation einzustellen.

Kurz nach Fortsetzung des Prozesses und unter Vermittlung von UN-Generalsekretär Kofi Annan verpflichteten sich die Kläger sechs Wochen später auf die vergleichsweise Rücknahme der Klage. Die südafrikanische Regierung erklärte im Gegenzug, die Regelungen des TRIPS-Übereinkommens bei der Umsetzung des Medicines Act zu beachten und die von geplanten Maßnahmen konkret betroffenen Pharmaunternehmen vorab zu konsultieren.

\section{Verfassungsrechtliche Bewertung}

Die folgende Bewertung der gegen den Amendment Act vorgetragenen verfassungsrechtlichen Bedenken konzentriert sich auf die durch das Verfahren 4183/98 aufgeworfenen grundrechtlichen Fragestellungen. ${ }^{91}$ Der Vorwurf, die im Gesetz enthaltenen Verordnungsermächtigungen (delegated legislation) seien zu unbestimmt, wird hierbei nicht näher beleuchtet. Angemerkt sei lediglich, dass Grundrechtseingriffe in Südafrika nach der ersten Voraussetzung der allgemeinen Schrankenbestimmung (limitation clause, Section 36 Constitution Act) ${ }^{92}$ nur durch oder aufgrund eines allgemeinen Gesetzes zulässig sind (threshold test). ${ }^{93}$ Aus dieser Bestimmung, die sich an Art. 19 Abs. 1 GG orientiert, ${ }^{94}$ wird unter Heranziehung des englischen Grundsatzes der rule of law gefolgert, dass ein grund-

89

90

91

92

Vgl. hierzu den Bericht in Guardian Unlimited vom 18.4.2001 unter http://www.guardian.co.uk/ aids/story/0,7369,474361,00.html.

Zum Vergleich vgl. die Pressemitteilung von GlaxoSmithKline vom 19.4.2001 (zu finden unter http://corp.gsk.com/press_archive/press_04192001.html).

Die Prüfung von Grundrechtseingriffen erfolgt in Südafrika nach dem Schema (1) Schutzbereich des betroffenen Grundrechts; (2) Eingriff in den Schutzbereich; (3) Grundrechtsschranken; vgl. Woolman, Limitation, in: Chaskalson et al., Constitutional Law in South Africa (Stand: Juni 1999), S. $12-2$ f.

Section 36 Constitution Act (limitation clause) erklärt: ,(1) The rights in the Bill of Rights may be limited only in terms of law of general application to the extent that the limitation is reasonable and justifiable in an open and democratic society based on human dignity, equality and freedom, taking into account all relevant factors, including - (a) the nature of the right; (b) the importance of the purpose of the limitation; (c) the nature and extent of the limitation; (d) the relation between the limitation and its purpose; and (e) less restrictive means to achieve the purpose. (2) Except as provided in subsection (1) or in any other provision of the Constitution, no law may limit any right entrenched in the Bill of Rights".

Die Eigentumsgarantie in Section 25 Constitution Act wiederholt diese Voraussetzung.

Woolman, a.a.O. (Fn. 91), S. 12-12. 
rechtseinschränkendes Gesetz u.a. hinreichend präzise gefasst sein muss. ${ }^{95}$ Dies setzt eine gewisse Regelungsdichte voraus, wobei vorgeschlagen worden ist, die Kriterien von Art. 80 Abs. 1 GG sowie die Wesentlichkeitsrechtsprechung des Bundesverfassungsgerichts bei der Auslegung der allgemeinen Schrankenbestimmung zu berücksichtigen. ${ }^{96}$ Darüber hinaus könnte auch Section 33 (1) (a) Constitution Act (just administrative action) Anforderungen an die Regelungsdichte von Verordnungsermächtigungen stellen. ${ }^{97}$ Angesichts der Intensität möglicher Grundrechtsbeeinträchtigungen aufgrund von Section 15C Medicines Act (im Extremfall kann der Gesundheitsminister die patentrechtlichen Schutzvorschriften für pharmazeutische Produkte durch Verordnung weitgehend aushöhlen) und dem offenen Wortlaut der Bestimmung, die auf more affordable medicines in certain circumstances abzielt, ist jedenfalls fraglich, ob diese Verordnungsermächtigung nach Inhalt, Zweck und Ausmaß tatsächlich hinreichend bestimmt ist. Dies gilt auch für Section 1 (4) Medicines Act (international tendering). Die Ermächtigung zur Einführung von Preiskontrollen und einheitlichen Gebühren für die Abgabe von Medikamenten in Section 22G (2) und (3) Medicines Act gibt dem Gesundheitsminister demgegenüber einen relativ detaillierten Rahmen vor. Schließlich begegnet die im Medicines Act selber genau vorgeschriebene bevorzugte Abgabe von Generika (generic subsitution) jedenfalls in Hinblick auf den threshold test von Section 36 (1) Constitution Act keinen verfassungsrechtlichen Bedenken.

Eine weitere Vorbemerkung gilt der Klagebefugnis. Der High Court hatte gleich zu Beginn der mündlichen Verhandlung im März 2001 angedeutet, dass die PMA und die klagenden Pharmaunternehmen durch den Amendment Act möglicherweise nicht in ihren Rechten betroffen sind, da die zur Umsetzung der angegriffenen Bestimmungen notwendigen Verordnungen noch nicht erlassen wurden und es dementsprechend auch an administrativen

Woolman, a.a.O. (Fn. 91), S. 12-28: ,This threshold test for permitting government justification of its actions is designed to do two things. First, it is intended to promote and give effect to the rule of law. Secondly, it should filter out bills of attainder. Laws under a regime governed by the rule of law have several notable attributes. First, under such a regime the law is supreme and general. (...) Secondly, the rule of law requires the creation of a positive body of law that permits citizens to know what the law expects of them. This requirement means that the law must be accessible to the citizenry - publicly promulgated laws only. Policies kept in a government drawer will not suffice. This second requirement also means that the law must be precise enough to permit individuals to plan their lives so as not to fall foul of the law. Laws, on this account, may not simply grant officials the discretion to use their power however they wish within a particular social sphere." Vgl. auch Erasmus, Limitation and Suspension, in: van Wyk / Dugard / de Villiers / Davis, Rights and Constitutionalism, 1994, S. 654.

de Ville, Interpretation of the general limitation clause in the chapter on fundamental rights, South African Public Law (SAPL) 1994, 287, 294; Rautenbach, General provisions of the South African bill of rights, 1995 , S. 88 .

Klaaren, Administrative Justice, in: Chaskalson et al., Constitutional Law of South Africa (Stand: Juni 1999), S. 25-13. Section 33 (1) (a) Constitution Act erklärt: „Everyone has the right to administrative action that is lawful, reasonable and procedurally fair". 
Entscheidungen bezüglich der Erteilung von Einfuhr- oder Produktionsgenehmigungen für Konkurrenten oder der Einführung von Preiskontrollen fehle. ${ }^{98}$ Hierzu ist anzumerken, dass jedenfalls die im Gesetz selbst vorgeschriebene bevorzugte Abgabe von Generika in Hinblick auf das Gleichbehandlungsgebot grundrechtsrelevant ist. Darüber hinaus eröffnet Section 38 Constitution Act in Südafrika die Möglichkeit von Popular- ${ }^{99}$ und Verbandsklagen. ${ }^{100}$ Der personale Schutzbereich der Grundrechte umfasst dabei grundsätzlich auch juristische Personen. ${ }^{101}$

Die ausschließliche Befugnis, pharmazeutische Erfindungen durch die Einfuhr oder Herstellung von Medikamenten zu nutzen, fällt in Südafrika als intellectual property in den Schutzbereich der Eigentumsgarantie (Section 25 Constitution Act). ${ }^{102}$ Aufgrund des geänderten Medicines Act sind erhebliche Einschnitte in diesen patentrechtlichen Schutz pharmazeutischer Produkte und Herstellungsverfahren möglich; die Erteilung von Lizenzen für die Einfuhr und Produktion von Generika an Konkurrenten von Patentinhabern (compulsory licensing) und Genehmigungen zum Import von in Südafrika patentierten Markenarzneimitteln aus Ländern, in denen diese Produkte billiger angeboten werden (parallel importation), hebt die ausschließliche Nutzungsbefugnis von Patentinhabern auf und greift damit in den Schutzbereich von Section 25 Constitution Act ein. Unabhängig von der patentrechtlichen Situation gilt dies auch für die Regulierung von Verkaufspreisen durch staatliche Preiskontrollen. Dabei ist zu berücksichtigen, dass compulsory licensing auch im pharmazeutischen Bereich schon vor den Änderungen des Medicines Act aufgrund patentrechtlicher Vorschriften (Sections 56 und 4 Patents Act ${ }^{103}$ ) möglich war; ${ }^{104}$ mit dem Amendment Act wurde allerdings eine Verordnungsermächtigung geschaffen, die über das patentrechtliche Einzelgenehmigungsverfahren hinausgeht und im Gegensatz zur Generalklausel in Section 56 Patents Act mit der medizinischen Versorgung der Bevölkerung ein ganz bestimmtes öffentliches Interesse verfolgt.

Vgl. hierzu den Bericht über den ersten Verhandlungstag von Independent Online vom 3.5.2001 unter http://www.iol.co.za/general/newsprint.php3?art_id=qw983806621565B263. Tatsächlich hat CIPLA, ein indischer Hersteller von Generika, erst nach Rücknahme der Klage den ersten Antrag auf die Erteilung einer Ausnahmelizenz gestellt.

99

Section 38 (d) Constitution Act.

100

Section 38 (e) Constitution Act.

101 Section 8 (4) Constitution Act.

102

Chaskalson / Lewis, Property, in: Chaskalson et al., Constitutional Law in South Africa (Stand: Juni 1999), S. 31-3 und 31-5 f.

103 104

Zu finden unter http://www.cptech.org/ip/health/sa/patlaw.html.

Der Patents Act wurde erst 1997 mit dem Intellectual Property Laws Amendment Act, Act No. 38 of 1997 geändert, um das Patentrecht in Übereinstimmung mit dem TRIPS-Übereinkommen zu bringen; vgl. Republic of South Africa, Government Gazette No. 18325 vom 1.10.1997, S. 2 (zu finden unter http://www.gov.za/acts/1997/a38.pdf). 
Grundrechtseingriffe sind in Südafrika nur zulässig, soweit sie verhältnismäßig sind. Dabei verlangt Section 36 Constitution Act, dass Eingriffe reasonable and justifiable in an open and democratic society based on human dignity, equality and freedom sind. Als zusätzliche Wertungskriterien benennt die Verfassung in Anlehnung an die Rechtsprechung des südafrikanischen Verfassungsgerichts ${ }^{105}$ fünf Faktoren, die bei der Überprüfung von Grundrechtseinschränkungen zu berücksichtigen sind: (a) das Wesen des durch die Einschränkung betroffenen Grundrechts; (b) die Bedeutung des hinter der Einschränkung stehenden Zwecks; (c) die Art und Weise sowie das Ausmaß der Grundrechtseinschränkung; (d) das Verhältnis zwischen Grundrechtseinschränkung und Zweck, sowie (e) die Berücksichtigung weniger einschneidender Alternativen. ${ }^{106}$ Der Staat muss hiernach mit dem Eingriff einen hinreichend wichtigen Zweck mit geeigneten und vertretbaren Mitteln verfolgen und dabei möglichst schonend mit den betroffenen Grundrechten umgehen. Zusätzlich verbietet Section 25 (1) Constitution Act willkürliche Eingriffe in das Eigentum (arbitrary deprivation of property).

Angesichts der aktuellen Aids-Situation in Südafrika und der greifbaren Folgen der Krankheit für das Leben und die Gesundheit vieler Menschen kann nun nicht ernsthaft bezweifelt werden, dass das mit dem Amendment Act verfolgte legislative Ziel jedenfalls in Bezug auf Aids-Medikamente die mit Section 36 Constitution Act aufgestellte Hürde überwindet. Die Zulassung von Generika und parallel importation bewirken erfahrungsgemäß erhebliche Preissenkungen auf dem nationalen Markt, ${ }^{107}$ die allerdings nur dann zu einer spürbaren Verbesserung der medizinischen Versorgung beitragen, wenn ein Gesundheitssystem auch technisch und administrativ in der Lage ist, die entsprechenden Medikamente wirklich zu den Betroffenen zu bringen. Die jüngsten Reformen des südafrikanischen Gesundheitswesens, die beispielsweise den Kreis der zur Abgabe von Medikamenten berechtigten Personen erweitern, lassen jedoch erwarten, dass diese Rahmenbedingungen in den kommenden Jahren jedenfalls sukzessive verbessert werden. Da der Staat bei der hohen Zahl von Betroffenen erkennbar nicht in der Lage ist, selbst deutlich reduzierte Preise für patentierte Markenarzneimittel zu zahlen, gibt es auch kaum weniger einschränkende Alternativen. Das Preisniveau kann für bestimmte Medikamente zwar auch durch ein staatliches Kontrollsystem gesenkt werden, für sich genommen stellen Preiskontrollen aber weder die inländische Produktion (notfalls, wie in Brasilien, durch staatliche Unternehmen) noch den Import von Medikamenten sicher. Erst die Öffnung des Marktes für andere Anbieter oder staatliche Unternehmen garantiert eine billige und zudem ausreichende Versorgung. Andererseits ist zu berücksichtigen, dass mit der angestrebten Marktöffnung die Rechte der Patentinhaber in ihrem Kernbestand betroffen sind; der Verlust ausschließlicher Nutzungs-

Sv Makwanyane \& another 1995 (6) BCLR 665 (CC) unter 708E-F.

106 Section 36 (1) (a) - (e) Constitution Act.

107 ICASO, Compulsory Licencing and Parallel Importing (Background Paper, July 1999), vgl. Fn. 44. 
rechte führt trotz des (bislang) verhältnismäßig kleinen südafrikanischen Marktes zu erheblichen wirtschaftlichen Einbußen. Hier stellt sich die Frage, ob nicht zumindest eine zeitliche Begrenzung von compulsory licensing und parallel importation, wie sie beispielsweise auch in Section 56 (4) (c) Patents Act vorgeschrieben wird, ${ }^{108}$ verlangt werden muss.

Eine weitere Problematik der Novelle ist der breite Anwendungsbereich der im Verordnungswege möglichen Ausnahmebestimmungen. Section 15C Medicines Act ist nicht auf Aids-Medikamente beschränkt, so dass der Gesundheitsminister den patentrechtlichen Schutz aller am südafrikanischen Markt angebotenen pharmazeutischen Produkte einschränken kann. Eine besondere Notlage in spezifischen Bereichen der medizinischen Versorgung (der mit einer sektoral begrenzten Senkung der Arzneimittelpreise begegnet werden könnte) wird nicht verlangt. Hier - wie auch bei der eben angesprochenen Intensität der Grundrechtseinschränkung durch den Amendment Act - gerät die verfassungsrechtliche Verankerung sozio-ökonomischer Rechte in den Blick. Section 27 Constitution Act enthält ein individuelles Grundrecht auf Zugang zu medizinischer Versorgung und stellt den Staat unter die ausdrückliche Pflicht, diesen Anspruch durch legislative und sonstige Maßnahmen zur Durchsetzung zu verhelfen; außerdem darf niemandem die erforderliche medizinische Notversorgung verwehrt werden. ${ }^{109}$ Interessant ist im vorliegenden Zusammenhang auch Section 7 (2) Constitution Act. Nach dieser - dem Grundrechtskatalog vorangestellten - Norm ist der Staat nicht nur verpflichtet, die Grundrechte zu beachten (hier findet der status negativus der Grundrechtsträger seinen Ausdruck); er ist vielmehr auch gehalten, die Grundrechtsträger zu schützen und aktiv auf die Realisierung der Grundrechte hinzuarbeiten. ${ }^{110}$ Die Staatszielbestimmung in Section 27 (2) Constitution Act wiederholt diese Verpflichtung u.a. in Bezug auf health care services, stellt das Handlungsprogramm des Staates aber zugleich auch unter den Vorbehalt ausreichender Ressourcen. ${ }^{111}$ Ungeachtet der schwierigen Frage, ob Betroffene in Südafrika aus Section 27 (1) und (2) Constitution Act subjektive Ansprüche auf eine verfassungsrechtlich garantierte medizinische Mindestversorgung (minimum care) herleiten können ${ }^{112}$ oder - etwa als Aids-Patienten - vom Staat

108

Section 56 (4) (c) Patents Act erklärt: „A licence granted under this section shall include a provision that, subject to adequate protection of the legitimate interest of the licensee, the licence shall, on application by the patentee, be terminated if the circumstances which led to its grant cease to exist and, in the opinion of the commissioner, are unlikely to recur".

Zum Wortlaut von Section 27 Constitution Act vgl. Fn. 79.

110 Section 7 (2) Constitution Act erklärt: „The state must respect, protect, promote and fulfil the rights in the Bill of Rights".

111 Das Verfassungsgericht hat dem Staat mit Rücksicht auf die weitreichenden Folgen individueller Leistungsansprüche auf den Staatshaushalt hierbei einen weiten Entscheidungsspielraum eingeräumt; vgl. Soobramoney v Minister of Health, KwaZulu-Natal 1997 (12) BCLR 1696 (CC).

So z. B. Liebenberg, Socio-economic Rights, in: Chaskalson et al., Constitutional Law in South Africa (Stand: Juni 1999), S. 41-43: „However, I would argue that a minimum core obligation (...) is also implicit in ss 26 and 27. In terms of this obligation the state must ensure that groups in 
zumindest auf Grundlage von Section 27 (3) Constitution Act notversorgt werden müssen, ${ }^{113}$ geben diese Bestimmungen dem gesetzgeberischen Zweck des Amendment Act Verfassungsrang und sind jedenfalls bei der Anwendung der Schrankenbestimmung zu berücksichtigen. ${ }^{114}$ Zusammen mit dem Recht auf Leben (Section 11 Constitution Act) ${ }^{115}$ und der Verpflichtung des Staates, die Menschenwürde zu schützen (Section 10 Constitution Act), ${ }^{116}$ spricht eine Abwägung zwischen den ökonomischen Interessen von Inhabern medizinischer Patente und dem öffentlichen Interesse an einer Versorgung der Bevölkerung nicht nur mit lebensnotwendigen Aids-Medikamenten für den Eingriff in die Eigentumsgarantie. Zwar ist die Situation der Aids-Patienten dabei besonders kritisch; die südafrikanische Regierung hat aber wiederholt deutlich gemacht, dass das medizinische System angesichts der Kosten für Pharmazeutika auch bei vielen anderen Krankheiten - wie etwa Tuberkulose - weit überfordert ist. ${ }^{117}$ Der Vorwurf einer verfassungswidrigen Enteignung geht ebenfalls fehl, da die südafrikanische Verfassung deutlich zwischen der entschädigungslosen Inhaltsbestimmung - deprivation of property, Section 25 (1) Constitution Act und der entschädigungspflichtigen Enteignung - expropriation of property, Section 25 (2) Constitution Act - unterscheidet. Letztere setzt die vollständige oder teilweise Entziehung vermögenswerter Rechtspositionen durch einen gezielten hoheitlichen Rechtsakt voraus (,not only dispossession or deprivation but also appropriation by the expropriator of the particular right, and abatement or extinction, as the case may be, of any other existing right

especially vulnerable and disadvantaged circumstances have access to a basic level of the socioeconomic rights. This should be sufficient to preserve human life and dignity." Vgl. auch de Vos, Pious Wishes or Directly Enforceable Human Rights?: Social and Economic Rights in South Africa's 1996 Constitution, South African Journal on Human Rights (SAJHR) 1997, 67, 87.

In der Entscheidung Soobramoney v Minister of Health, KwaZulu-Natal 1997 (12) BCLR 1696 (CC) hat das Verfassungsgericht die vom Kläger beanspruchte Dialyse-Behandlung nicht unter die von Section 27 (3) Constitution Act gewährleistete emergency medical treatment gefasst. Der Kläger verstarb kurz nach der Entscheidung.

114 Liebenberg, a.a.O. (Fn. 112), S. 41-50: „Finally, the state's duty to realize a socio-economic right may constitute an important purpose for limiting other rights in the Bill of Rights. For example, it may be reasonable and justifiable to limit the property rights in s 25(2) and (3) in order to achieve equitable access to water in South Africa. The degree of permissible limitation will depend on the application of the proportionality test under the limitation clause".

115 Nach du Plessis / de Ville, Personal Rights: Life, Freedom and Security of the Person, Privacy and Freedom of Movement, in: van Wyk / Dugard / de Villiers / Davis, Rights and Constitutionalism, 1994, S. 221 f. könnte das Recht auf Leben subjektive Ansprüche auf medizinische Versorgung begründen. Ebenso Fedler, Life, in: Chaskalson et al., Constitutional Law in South Africa (Stand: Juni 1999), S. 15-3 f.

116 Nach Basson, South Africa's Interim Constitution, 2. Aufl., 1995, S. 24/25, leitet sich aus der Menschenwürde jedenfalls eine Staatszielbestimmung ab, die auch eine medizinische Mindestversorgung umfaßt.

Zum Beispiel Gesundheitsministerin Tschabalala-Msimang in Business Day, Johannesburg, zu finden unter http://www.bday.co.za/bday/content/direct/1,3523,832452-6096-0,00.html 
held by another which is inconsistent with the appropriated right “" ${ }^{118}$ ). Diese Voraussetzungen erfüllen die angegriffenen Einschränkungen des Patentrechts nicht.

Der zweite hier zu behandelnde Vorwurf gegenüber dem Amendment Act betrifft die Berufsfreiheit, die von Section 22 Constitution Act gewährleistet wird. Interessanterweise orientiert sich diese Verfassungsvorschrift eng am Grundgesetz und differenziert wie Art. 12 Abs. 1 GG zwischen Berufswahl (right of choice) und Berufsausübung (regulation of practice). ${ }^{119}$ Die Verpflichtung zur vorrangigen Abgabe von Generika (generic substitution) und die Einführung von einheitlichen Abgabegebühren für Medikamente sind Regelungen zur Berufsausübung und greifen daher in den Schutzbereich von Section 22 Constitution Act ein. Der verfassungsrechtliche Prüfungsmaßstab für diese Eingriffe ist wieder der soeben erörterten allgemeinen Schrankenbestimmung zu entnehmen, wobei sowohl der Wortlaut von Section 22 Constitution Act als auch eine historische Auslegung der Verfassungsbestimmung eine abgestufte Schutzintensität zwischen Berufswahl und Berufsausübung nahe legen. ${ }^{120}$ Während die Berufswahl in Section 22 Constitution Act zunächst schrankenlos gewährt wird und somit nur der allgemeinen Schrankenbestimmung unterliegt, steht die Berufsausübung schon nach dem Wortlaut der grundrechtlichen Gewährleistung selbst unter einem Gesetzesvorbehalt (,the practice of a trade, occupation or profession may be regulated by law"). Dem Gesetzgeber wird damit ein größerer Spielraum bei der Ausgestaltung der Berufsausübung eingeräumt. Es wird sogar vorgeschlagen, die Prüfung von Maßnahmen zur regulation of practice auf Section 22 Constitution Act zu begrenzen und lediglich Einschränkungen der Berufswahl an der Verhältnismäßigkeitsschranke von Section 36 Constitution Act zu messen. Dies würde praktisch auf eine Prüfung der Geeignetheit hinauslaufen (,,a rational connection between the regulatory statute and the objective sought to be achieved"), ${ }^{121}$ wobei die in Section 26 (2) der Übergangsverfassung von 1993/1994 ${ }^{122}$ enthaltenen Kriterien als Maßstab für Grundrechtseinschrän-

Beckenstrater v Sand River Irrigation Board 1964 (4) SA 510 (T) unter 515B-C; Chaskalson / Lewis, a.a.O. (Fn. 102), S. 31-15: „,... its legal meaning now includes an element of acquisition“.

Section 22 Constitution Act erklärt: „Every citizen has the right to choose their trade, occupation or profession freely. The practice of a trade, occupation or profession may be regulated by law“. Diese Verfassungsbestimmung weist deutliche Parallelen zu Art. 12 GG auf; vgl. Davis, Economic Activity, in: Chaskalson et al., Constitutional Law in South Africa (Stand: Juni 1999), S. 2916.

Davis, a.a.O. (Fn. 119), S. 29-17: „Within the South African context the infringement of choice should be examined rather as a limitation problem in terms of $\mathrm{s} 36$, as opposed to a question about the regulation of practice, which falls for determination under s 22 “.

Section 26 Constitution of the Republic of South Africa, 1993, Act 200 of 1993 erklärt: „Every person shall have the right freely to engage in economic activity and to pursue a livelihood anywhere in the national territory. (2) Subsection (1) shall not preclude measures designed to promote the protection or the improvement of the quality of life, economic growth, human development, social justice, basic conditions of employment, fair labour practices or equal opportunity for all, 
kungen herangezogen werden könnten. ${ }^{123}$ Da die allgemeine Schrankenbestimmung jedoch ausdrücklich alle Grundrechtsbeschränkungen erfasst, erscheint eine so weitgehende Herabsetzung des Schutzniveaus nicht mehr verfassungsgemäß.

Unter Zugrundelegung der bereits dargelegten verfassungsrechtlichen Pflicht des Staates, eine angemessene medizinische Versorgung für alle Teile der Bevölkerung sicherzustellen (Section 27 (2) Constitution Act), und der Bedeutung des Amendment Act für die in vielen Bereichen dringend notwendige Senkung der Kosten im Gesundheitswesen erscheint die Verpflichtung von Apothekern und anderen zur Abgabe von Medikamenten berechtigten Personen, ihren Kunden vorrangig Generika anzubieten (was nicht ausschließt, dass sich Patienten dann trotzdem für teure Markenarzneimittel entscheiden) als eine verhältnismäßige Regelung der Berufsausübung. Dabei ist auch zu berücksichtigen, dass Apotheken in Südafrika sehr häufig von den behandelnden Ärzten selbst betrieben werden, so dass eine Trennung zwischen einer möglichst objektiven medizinischen Beratung der Patienten und einer wirtschaftlichen Betätigung durch den Verkauf von Medikamenten, die in anderen Systemen durch Berufsinkompatibilitäten sichergestellt wird, nicht besteht. ${ }^{124}$ Dieselben Argumente streiten auch für die Verfassungsmäßigkeit von einheitlichen dispensing fees. Dabei ist zu berücksichtigen, dass Section 22G (2) (b) Medicines Act ausdrücklich die Festlegung angemessener Rezeptgebühren verlangt, so dass das hierfür zuständige Pricing Committee auch die wirtschaftliche Existenz des betroffenen Personenkreises als Kriterium zu beachten haben wird.

Schließlich ist das aus Section 9 Constitution Act folgende Gleichbehandlungsgebot der Hersteller betroffen, wenn Apotheker und andere zur Abgabe von Medikamenten berechtigte Personen durch die Vorschriften über generic substitution verpflichtet werden, an ihre Kunden vorrangig Generika abzugeben. Dabei genügt seit der Prinsloo-Entscheidung des südafrikanischen Verfassungsgerichts ${ }^{125}$ im Bereich des allgemeinen Gleichheitssatzes auf einer ersten Prüfungsstufe - der infringement inquiry stage, Section 9 (1) Constitution Act $^{126}$ - jeder sachliche Differenzierungsgrund (rational connection to a legitimate government purpose), um Ungleichbehandlungen zu rechtfertigen (Willkürverbot). Ungleichbehandlungen, die zu einer Diskriminierung aufgrund der in Section 9 (3) Constitu-

provided such measures are justifiable in an open and democratic society based on freedom and equality".

123 So im Ansatz die Eastern Cape Provincial Division des High Court in SA Post Office Ltd $v$ Van Rensburg \& another 1998 (1) SA 796 (E) unter 805G.

124

Vgl. hierzu den Bericht in Guardian Unlimited vom 5.3.2001 (zu finden unter http://www.

125 guardianunlimited.co.uk/international/story/0,3604,446465,00.html).

126

Prinsloo $v$ Van der Linde and another 1997 (6) BCLR 759 (CC).

Section 9 (1) Constitution Act erklärt: „Everyone is equal before the law and has the right to equal protection and benefit of the law". 
tion Act $^{127}$ benannten Kriterien führen oder die Menschenwürde beeinträchtigen, sind dabei trotz Vorliegens eines sachlichen Grundes zwar grundsätzlich verboten (unfair discrimination stage), können aber wiederum unter den Voraussetzungen der allgemeinen Schrankenbestimmung gerechtfertigt sein.

Das mit generic substitution verfolgte gesetzgeberische Ziel, Patienten über die zur Verfügung stehenden kostengünstigen Generika zu informieren und zu einem bevorzugten Einsatz dieser Mittel zu gelangen, wenn nicht der Patient widerspricht oder aber generic substitution (im konkreten Einzelfall) durch den verschreibenden Arzt oder (generell) durch die zuständige Aufsichtsbehörde ausdrücklich ausgeschlossen worden ist, führt zu einer Benachteiligung von Markenarzneimitteln. Die Anbieter von Generika gewinnen auf diesem Weg ohne eigene Anstrengungen Marktanteile, während die Hersteller von Markenarzneimitteln im Wettbewerb einem erhöhten Begründungszwang ausgesetzt werden. Das einzige Differenzierungsmerkmal ist dabei der Verkaufspreis. Soweit in erster Linie der Staat über das öffentliche Gesundheitssystem die Kosten der verschriebenen Pharmazeutika am Ende tragen muss und über eine Senkung dieser Ausgaben eine bessere medizinische Versorgung der Bevölkerung gewährleisten kann, wird dieses gesetzgeberische Anliegen jedenfalls die Anforderungen der infringement inquiry stage erfüllen. Unter Fürsorgegesichtspunkten erscheint dabei insbesondere die reine Informationspflicht gerechtfertigt, da viele Patienten über die Möglichkeit einer billigeren Behandlung mit Generika in ihrem konkreten Einzelfall nicht informiert sein werden; diese Informationspflicht könnte - alternativ - auch schon bei den Ärzten ansetzen. Probleme wirft generic substitution allerdings dort auf, wo Patienten die Kosten ihrer Behandlung privat bezahlen, da der Staat hier (ohne Vorteile für die Allgemeinheit) in die wirtschaftliche Auseinandersetzung von Anbietern zugunsten ganz bestimmter Konkurrenten eingreift. Angesichts der geringen Anforderungen, die die Rechtsprechung in Südafrika an das allgemeine Gleichheitsgebot stellt, könnte aber selbst der Schutz des finanziell besser gestellten Patienten als gesetzgeberisches Anliegen ausreichen. Schließlich ist generic substitution unter Berücksichtigung der Staatszielbestimmung in Section 27 (2) Constitution Act wohl auch nicht als unfaire Diskriminierung zu qualifizieren. Dabei ist nach dem heutigen Stand der Rechtsprechung ohnehin nicht klar, ob Section 9 (3) Constitution Act auf juristische Personen überhaupt anwendbar ist, da die genannten Differenzierungsmerkmale ausschließlich an die Eigenschaften von natürlichen Personen geknüpft sind.

Section 9 (3) Constitution Act erklärt: „The state may not unfairly discriminate directly or indirectly against anyone on one or more grounds, including race, gender, sex, pregnancy, martial status, ethnic or social origin, colour, sexual orientation, age, disability, religion, conscience, belief, culture, language and birth." Diese Liste ist nicht abschließend, vgl. Kentridge, Equality, in: Chaskalson et al., Constitutional Law in South Africa (Stand: Juni 1999), S. 14-32B.

Vgl. zu diesem Problem Kentridge, a.a.O. (Fn. 127), S. 14-47 f. 
Als Zwischenergebnis ist an dieser Stelle somit festzuhalten, dass der Amendment Act jedenfalls in Hinblick auf die Eigentumsgarantie, die Freiheit der Berufsausübung sowie das Gleichbehandlungsgebot nach südafrikanischem Recht verfassungsgemäß ist.

\section{Der Medicines Act und TRIPS}

Die Republik Südafrika hat (im Gegensatz zu vielen anderen Entwicklungs- und Schwellenländern) bereits seit einigen Jahren relativ strenge Patentgesetze und ist inzwischen allen wichtigen internationalen Abkommen zum Patentschutz beigetreten. Hierzu gehört auch das zwischen 1986 und 1994 im Rahmen der Uruguay-Runde ausgehandelte TRIPS-Übereinkommen der WTO, an dessen Vorschriften Südafrika seit 1995 uneingeschränkt gebunden ist. ${ }^{129}$ Bei der Überprüfung des Amendment Act anhand der Bestimmungen des Übereinkommens ist jedoch $\mathrm{zu}$ berücksichtigen, dass dieses internationale Instrument kein einheitliches und unmittelbar anwendbares Gesetz darstellt, sondern lediglich den Mindeststandard für einen patentrechtlichen Schutz definiert, der von den Unterzeichnerstaaten durch nationales Recht zu gewährleisten ist. ${ }^{130}$ Ungeachtet der Frage, ob die Beachtung des TRIPS-Übereinkommens durch den Gesetzgeber in Südafrika - etwa von Pharmaunternehmen - auf Grundlage der nationalen Verfassung klagweise eingefordert werden kann (das Übereinkommen selbst gewährt Patentinhabern keinen subjektiven Schutz und sieht lediglich Mechanismen zur internationalen Streitvermeidung und -beilegung vor ${ }^{131}$ ), soll hier die Zulässigkeit von Parallelimporten und Zwangslizenzen geprüft sowie der Frage nach der Erforderlichkeit einer Entschädigung für Eingriffe in das nationale Patentrecht nachgegangen werden.

Das TRIPS-Übereinkommen bildet neben dem Protokoll von Marrakesch zum Allgemeinen Zoll- und Handelsübereinkommen (GATT) ${ }^{132}$ und dem Allgemeinen Übereinkommen über

Vielen WTO-Mitgliedern wurden mit Rücksicht auf ihren Entwicklungsstand Übergangsfristen bei der Anpassung ihres nationalen Rechts eingeräumt. Alle WTO-Mitglieder müssen spätestens ab 2005/2006 einen 20-jährigen Patentschutz für pharmazeutische Erfindungen gewähren.

130 Art. 1 (1) TRIPS-Übereinkommen erklärt: „Die Mitglieder wenden die Bestimmungen dieses Übereinkommens an. Die Mitglieder dürfen in ihr Recht einen umfassenderen Schutz als den durch dieses Übereinkommen geforderten aufnehmen, vorausgesetzt, dieser Schutz läuft diesem Übereinkommen nicht zuwider, sie sind dazu aber nicht verpflichtet. Es steht den Mitgliedern frei, die für die Umsetzung dieses Übereinkommens in ihrem eigenen Rechtssystem und in ihrer Rechtspraxis geeignete Methode festzulegen“.

131 Art. 63 f. TRIPS-Übereinkommen.

132 Das GATT (General Agreement on Tariffs and Trade) ist eine 1947 gegründete internationale Wirtschaftsorganisation. GATT ist nunmehr einbezogen in das umfassende Vertragswerk der WTO. 
den Handel mit Dienstleistungen (GATS) ${ }^{133}$ die dritte Säule der WTO. Es setzt relativ hohe Mindeststandards für den geistigen Eigentumsschutz in den wirtschaftlich wichtigsten Schutzbereichen ${ }^{134}$ und stärkt die tatsächliche Durchsetzung dieser Rechte durch detaillierte Verfahrensrichtlinien. ${ }^{135}$ Im Bereich des Patentrechts regelt das TRIPS-Übereinkommen dabei verbindlich, welche Gegenstände unter welchen allgemeinen Voraussetzungen patentiert werden müssen (Art. 27 und 29), welche Wirkungen Patente zu entfalten haben (Art. 28), wie lange die Schutzdauer sein muss (Art. 33) sowie Fragen der Beweislast (Art. 34). Ferner schreibt das Übereinkommen den WTO-Mitgliedsstaaten vor, unter welchen Voraussetzungen sie ausnahmsweise ohne Zustimmung des Patentinhabers die Benutzung einer patentierten Erfindung erlauben (Art. 30 und 31) oder Erfindungen gänzlich von der Patentierbarkeit ausschließen dürfen (Art. 27 Abs. 2 und 3). Insgesamt führen die Regelungen damit zu einer internationalen Rechtsangleichung auf dem Gebiet des Patentrechts, verschärfen diesen Schutz aber zugleich auch in den meisten Entwicklungsländern, da hier häufig keine oder doch nur lückenhafte Bestimmungen existieren. ${ }^{136}$ Es erweitert den Patentschutz insbesondere auf pharmazeutische Produkte und verschärft die nach den Regelungen der Pariser Verbandsübereinkunft zum Schutz des gewerblichen Eigentums (PVÜ) geltenden Rechtslage, unter der mehr als die Hälfte der PVÜ-Verbandsstaaten keinen Patentschutz in diesem Bereich gewähren. ${ }^{137}$ Obwohl das TRIPS-Übereinkommen Übergangsbestimmungen vorsieht, die die wirtschaftlichen Auswirkungen für unterentwickelte Länder (least developed countries) durch längere Anpassungszeiträume abmildern sollen, ${ }^{138}$ und in bestimmten Härtesituationen (etwa zum Schutz der öffentlichen Ordnung, der Gesundheit oder für medizinische Verfahren) Ausnahmen vom geforderten Schutzniveau erlaubt, ist das Regelwerk seit seiner Verabschiedung im Jahr 1994 stark kritisiert worden. Dabei wird befürchtet, dass sich der für die wirtschaftliche Entwicklung vieler Länder notwendige Technologietransfer verlangsamen könnte. ${ }^{139}$ Darüber hinaus wollte die Gruppe der Entwicklungsländer (angeführt durch Brasilien und Indien) insbesondere phar-

34 Urheberrecht und verwandte Schutzrechte (Art. 9 ff.), Marken (Art. 15 ff.), geographische Angaben (Art. 22 ff.), Patente (Art. 27 ff.), Layout-Design integrierter Schaltkreise (Art. 35 ff.) und Geschäftsgeheimnisse (Art. 39).

Art. 41 ff. Vgl. hierzu Dreier, TRIPS und die Durchsetzung von Rechten des geistigen Eigentums, GRUR Int. 1996, 205 ff.

136

137

Straus, a.a.O. (Fn. 39), S. 179, 181 f.; Pacón, a.a.O. (Fn. 42), S. 885 f.

38 Art. 65 f. und - speziell für pharmazeutische und agrochemische Erzeugnisse - Art. 70 Abs. 8 und 9; vgl. hierzu Straus, a.a.O. (Fn. 39), S. 204.

Die kritischen Stimmen zusammenfassend Hilpert, TRIPS und das Interesse der Entwicklungsländer am Schutz von Immaterialgüterrechten in ökonomischer Sicht, GRUR Int. 1998, 91, 92; Liebig, a.a.O. (Fn. 38). 
mazeutische Produkte und Nahrungsmittel vom internationalen Patentschutz ausnehmen. ${ }^{140}$ Die erforderliche Zustimmung zu dem Übereinkommen, das auf Initiative der US-amerikanischen Pharmaindustrie auf die Tagesordnung der Uruguay-Verhandlungen kam, wurde im Rahmen einer Paketlösung mit Zugeständnissen in anderen wichtigen Bereichen - vor allem beim Marktzugang für Agrar- und Textilprodukte - und den genannten (langen) Übergangsfristen trotzdem sichergestellt. ${ }^{141}$ Die Angst vor den Auswirkungen der TRIPSVerpflichtungen lässt Gesundheitsexperten vieler Unterzeichnerstaaten jetzt aber nach Auswegen suchen.

Eine wichtige Vorschrift in diesem Zusammenhang ist Art. 6 des TRIPS-Übereinkommens, wonach die Entscheidung, ob Importe von patentgeschützten Produkten legaler Lizenznehmer aus Drittländern zugelassen (Grundsatz der internationalen Erschöpfung von Rechten) oder aber verboten werden, nach allgemein herrschender Auffassung in das Ermessen des nationalen Gesetzgebers gestellt ist. ${ }^{142}$ WTO-Mitgliedsstaaten sind dabei nur an die Grundsätze der Inländerbehandlung ${ }^{143}$ und der Meistbegünstigung ${ }^{144}$ gebunden. Dies führt dazu, dass patentgeschützte Markenarzneimittel in einigen Ländern zu unterschiedlichen Preisen erhältlich sind - je nachdem, ob ein bestimmtes Produkt im Inland oder aber (von demselben Anbieter) im Ausland hergestellt und dann (von anderen Unternehmern oder staatlichen Stellen) importiert worden ist. ${ }^{145}$ Hinsichtlich der im Amendment Act vorgesehenen Bestimmungen zu parallel importation und international tendering

140

Zu den TRIPS-Verhandlungen vgl. Bail, Das Profil einer neuen Welthandelsordnung: was bringt die Uruguay-Runde? - Teil 2, EuZW 1990, 469 ff.; Heath, a.a.O. (Fn. 36), S. 1174 ff.; Pacón, a.a.O. (Fn. 42), S. 879; Straus, a.a.O. (Fn. 39), S. 187 ff.

141

Pacón, a.a.O. (Fn. 42), S. 876; Straus, a.a.O. (Fn. 39), S. 182 f.

142

So z.B. Pacón, a.a.O. (Fn. 42), S. 878; Heath, a.a.O. (Fn. 36), S. 1180 f.; sowie Ullrich, a.a.O. (Fn. 32), S. 623, 635. Art. 6 TRIPS-Übereinkommen erklärt: „Für die Zwecke der Streitbeilegung im Rahmen dieses Übereinkommens darf vorbehaltlich der Art. 3 und 4 dieses Übereinkommen nicht dazu verwendet werden, die Frage der Erschöpfung von Rechten des geistigen Eigentums zu behandeln“.

143

Art. 3 (1) 1 TRIPS-Übereinkommen erklärt: „Die Mitglieder gewähren den Angehörigen der anderen Mitglieder eine Behandlung, die nicht weniger günstig ist als die, die sie ihren eigenen Angehörigen in bezug auf den Schutz des geistigen Eigentums gewähren, vorbehaltlich der jeweils bereits in der Pariser Verbandsübereinkunft (1967), der Berner Übereinkunft (1971), dem RomAbkommen oder dem Vertrag über den Schutz des geistigen Eigentums im Hinblick auf integrierte Schaltkreise vorgesehenen Ausnahmen." $\mathrm{Zu}$ den Problemen der Entwicklungsländer mit dem Grundsatz der Inländerbehandlung vgl. Pacón, a.a.O. (Fn. 42), S. 877.

144

Art. 4 S. 1 TRIPS-Übereinkommen erklärt: „In bezug auf den Schutz des geistigen Eigentums werden Vorteile, Vergünstigungen, Sonderrechte und Befreiungen, die von einem Mitglied den Angehörigen eines anderen Landes gewährt werden, sofort und bedingungslos den Angehörigen aller anderen Mitglieder gewährt." Zu den Problemen der Entwicklungsländer mit dem Grundsatz 145 der Meistbegünstigung vgl. Pacón, a.a.O. (Fn. 42), S. 877 f.

Vgl. ICASO, Compulsory Licencing and Parallel Importing (Background Paper, July 1999), a.a.O. (Fn. 44). 
bestehen aus Sicht des TRIPS-Übereinkommens insofern keine Bedenken. ${ }^{146}$ Der Vollständigkeit halber ist jedoch anzumerken, dass Parallelimporte patentierter Erzeugnisse in Deutschland wie auch in den meisten anderen europäischen Staaten nach den nationalen Patentgesetzen nicht zulässig sind; die Erschöpfung von Patentrechten wird vielmehr von einem patentrechtlich zulässigen Inverkehrbringen des patentierten Erzeugnisses im Inland abhängig gemacht (Grundsatz der nationalen Erschöpfung von Rechten). ${ }^{147}$ Dabei steht dem Inverkehrbringen im Inland nach der Rechtsprechung des Europäischen Gerichtshofes das Inverkehrbringen in einem anderen Mitgliedsstaat der Europäischen Gemeinschaft gleich, wodurch wiederum Spielräume für Preisunterschiede entstehen.

Problematisch ist indes die Rechtslage bei der Frage nach compulsory licensing. Zwangslizenzen kommen im Rahmen von Art. 31 TRIPS-Übereinkommen insbesondere als Instrumente zur Bewältigung nationaler Notstände in Betracht. ${ }^{148}$ Dabei müssen - auch bei einer Benutzung durch die Regierung oder von der Regierung ermächtigte Dritte - im Wesentlichen folgende Voraussetzungen erfüllt sein: (1) die Erlaubnis zur Benutzung eines Patentgegenstandes ohne Zustimmung des Rechtsinhabers ist im Rahmen einer Einzelfallprüfung zu erteilen; ${ }^{149}$ (2) der Antragsteller muss sich bemüht haben, die Zustimmung des Rechtsinhabers zu angemessenen geschäftsüblichen Bedingungen zu erhalten, diese Bemühungen müssen jedoch innerhalb einer angemessenen Frist erfolglos geblieben sein; ${ }^{150}$ (3) Umfang und Dauer einer Benutzung sind auf den Zweck zu begrenzen, für den sie gestattet wurde; ${ }^{151}$ (4) eine Benutzung kann nur zusammen mit dem Teil des Unternehmens oder des Goodwill, dem diese Benutzung zusteht, übertragen werden; ${ }^{152}$ (5) eine Benutzung ist vorwiegend für die Versorgung des Binnenmarktes zu gestatten; ${ }^{153}$ (6) die Gestattung einer Benutzung ist vorbehaltlich eines angemessenen Schutzes der berechtigten Interessen der zu ihr ermächtigten Personen zu beenden, sofern und sobald die Umstände, die zu ihr geführt haben, nicht mehr vorliegen und wahrscheinlich nicht wieder eintreten werden; die zuständige Stelle muss die Befugnis haben, auf begründeten Antrag hin die Fortdauer dieser Umstände zu überprüfen; ${ }^{154}$ (7) dem Rechtsinhaber ist eine nach den Umständen des

146 Kritisch zu dieser Auslegung von Art. 6 TRIPS-Übereinkommen Straus, a.a.O. (Fn. 39), S. 193 ff.

Zu Deutschland vgl. die Entscheidungen BGHZ 49, 331 („Voran“) und BGH, GRUR 1976, 579 (,Tylosin“); rechtsvergleichend Beier, Zur Zulässigkeit von Parallelimporten patentierter Erzeugnisse, GRUR Int. 1996, 1, 3 ff.

$148 \mathrm{Zu}$ Art. 31 TRIPS-Übereinkommen vgl. Heath, a.a.O. (Fn. 36), S. 1175 f.

149

Art. 31 a) TRIPS-Übereinkommen.

150 Art. 31 b) TRIPS-Übereinkommen.

151 Art. 31 c) TRIPS-Übereinkommen

152 Art. 31 e) TRIPS-Übereinkommen.

153 Art. 31 f) TRIPS-Übereinkommen.

154 Art. 31 g) TRIPS-Übereinkommen. 
Falles angemessene Vergütung zu leisten, wobei der wirtschaftliche Wert der Erlaubnis in Betracht zu ziehen ist; ${ }^{155}$ (8) die Rechtsgültigkeit von Entscheidungen im Zusammenhang mit der Erlaubnis zu einer Benutzung und der vorgesehenen Vergütung unterliegen der Nachprüfung durch ein Gericht oder einer sonstigen unabhängigen Nachprüfung durch eine gesonderte übergeordnete Behörde. ${ }^{156}$ Auf das Erfordernis einer Zustimmung (bzw. ihrer Versagung) durch den Rechtsinhaber kann dabei verzichtet werden, wenn ein nationaler Notstand oder sonstige Umstände von äußerster Dringlichkeit vorliegen oder wenn es sich um eine öffentliche, nicht gewerbliche Benutzung handelt. ${ }^{157}$ Während sich Section 56 des südafrikanischen Patents Act nach den 1997 vorgenommenen Änderungen offensichtlich eng an diesen Voraussetzungen orientiert, erlaubt Section 15C Medicines Act die Erteilung von Zwangslizenzen im Verordnungswege ohne eine Bindung an die in Art. 31 TRIPSÜbereinkommen genannten Einschränkungen. Allenfalls das Zustimmungserfordernis aus Art. 31 b) TRIPS-Übereinkommen könnte dabei durch die Erklärung eines nationalen Notstandes - etwa hinsichtlich der Bedrohung durch Aids - in Zukunft überwunden werden (die Option, wegen der Seuche einen nationalen Notstand zu erklären, ist in Südafrika auch tatsächlich stark diskutiert worden); nur im Bereich der nicht-gewerblichen Nutzung durch staatliche Stellen ist die Zustimmung nicht erforderlich. Insgesamt erfüllt Section 15C Medicines Act in seiner derzeitigen Fassung aber erkennbar nicht die Anforderungen aus Art. 31 TRIPS-Übereinkommen, so dass die südafrikanische Regierung auf dieser Rechtsgrundlage ohne eine Verletzung internationaler Verpflichtungen derzeit nur begrenzte Ausnahmen von den Rechten aus Patenten, die nicht unangemessen im Widerspruch zur normalen Verwertung eines Patents stehen und die berechtigten Interessen des Patentinhabers nicht unangemessen beeinträchtigen, einführen kann. Dies erlaubt Art. 30 TRIPS-Übereinkommen. ${ }^{158}$ Weitergehende Einschränkungen durch die Erteilung von Zwangslizenzen können nur im Wege einer Einzelfallprüfung (und nicht im Verordnungswege) auf Antrag der Regierung oder Dritter auf der Grundlage von Section 56 Patents Act erwirkt werden. Dabei ist - wie auch von Art. 31 TRIPS-Übereinkommen gefordert - eine angemessene Vergütung an den Patentinhaber zu zahlen. ${ }^{159}$

155

Art. $31 \mathrm{~h}$ ) TRIPS-Übereinkommen.

157

Art. 31 b) TRIPS-Übereinkommen.

Art. 30 TRIPS-Übereinkommen erklärt: „Die Mitglieder können begrenzte Ausnahmen von den ausschließlichen Rechten aus einem Patent vorsehen, sofern solche Ausnahmen nicht unangemessen im Widerspruch zur normalen Verwertung des Patents stehen und die berechtigten Interessen des Inhabers des Patents nicht unangemessen beeinträchtigen, wobei auch die berechtigten Interessen Dritter zu berücksichtigen sind“"

Section 56 (7) Patents Act erklärt: „In determining the conditions on which any licence is granted the commissioner shall have regard to any relevant facts, including the risks to be undertaken by the licensee, the research and development undertaken by the patentee and the terms and conditions usually stipulated in licence agreements in respect of the subject-matter of the invention, between persons who voluntarily enter into such agreements“. 
Obwohl Art. 27 Abs. 2 TRIPS-Übereinkommen vorsieht, dass Mitglieder Erfindungen von der Patentierbarkeit ausschließen können, wenn die Verhinderung ihrer gewerblichen Verwertung innerhalb ihres Hoheitsgebiets zum Schutz der öffentlichen Ordnung oder der guten Sitten einschließlich des Schutzes des Lebens oder der Gesundheit von Menschen, Tieren oder Pflanzen oder zur Vermeidung einer ernsten Schädigung der Umwelt notwendig ist (Verwertungsverbote), können die nach dem Amendment Act zugelassenen Zwangslizenzen auch nicht auf diese Ausnahmevorschrift gestützt werden, da ja gerade eine intensivere gewerbliche Nutzung (und nicht ihr Ausschluss) durch die Förderung von billigen Konkurrenzprodukten vom südafrikanischen Gesetzgeber angestrebt wird. Solange der Vertrieb und Verkauf von bestimmten pharmazeutischen Produkten nach nationalem Recht erlaubt sind, ist ein Ausschluss dieser Produkte von der Patentierbarkeit nach Art. 27 Abs. 2 TRIPS-Übereinkommen nicht zulässig. ${ }^{160}$ Die Ausnahmeregelungen in Art. 27 Abs. 3 TRIPS-Übereinkommen erfassen dabei zwar diagnostische, therapeutische und chirurgische Verfahren für die Behandlung von Menschen oder Tieren, nicht aber Pharmazeutika.

\section{Schlussbemerkungen}

Die vorangegangene Bewertung des Medicines and Related Substances Control Amendment Act von 1997 hat gezeigt, dass die Änderungen des südafrikanischen Arzneimittelgesetzes in den meisten Punkten verfassungsgemäß sind. Die von den Pharmaunternehmen heftig kritisierten Parallelimporte verletzen auch nicht das internationale TRIPS-Übereinkommen, wohingegen die mit Section 15C Medicines Act eingeführte Verordnungsbefugnis zur Erteilung von Zwangslizenzen im Bereich pharmazeutischer Produkte bei einer Überprüfung im Rahmen eines Streitbeilegungsverfahrens vor der WTO sehr wahrscheinlich an den Anforderungen von Art. 31 TRIPS-Übereinkommen scheitern würde. In der Praxis ist dies unschädlich, da entsprechende Lizenzen auch auf der Grundlage des südafrikanischen Patentgesetzes erteilt werden können; Section 56 Patents Act erfüllt die TRIPSAnforderungen, verlangt allerdings auch die Zahlung einer Vergütung für die zwangsweise Benutzung einer Erfindung. Jenseits des Verfahrens 4183/98 des High Court in Pretoria zeigt das Beispiel Südafrika, dass sowohl die tatsächlichen als auch die verfassungsrechtlichen Gegebenheiten in vielen Entwicklungsländern in einem Spannungsverhältnis zum international angestrebten Patentschutz stehen. Dies muss etwa im Falle einer zwangsweisen Nutzung von pharmazeutischen Erfindungen (Art. 31 TRIPS-Übereinkommen) Auswirkungen auf die Höhe der fälligen Vergütung nach nationalem Recht haben. Die in vielen Sektoren katastrophale medizinische Versorgung der Bevölkerung in unterentwickelten Mitgliedsstaaten der WTO und der geringe finanzielle Spielraum der betroffenen Regierungen streiten aber auch für die ausdrückliche Zulassung von Parallelimporten im Rahmen einer künftigen Reform des TRIPS-Übereinkommens. Schließlich zeigt das sogenannte 


\begin{abstract}
„Aids-Verfahren“, dass die verfassungsrechtliche Abwägung zwischen der Sicherung einer angemessenen medizinischen Versorgung und Patentrechten in unterentwickelten Ländern nicht zuletzt aufgrund der Bedeutung sozio-ökonomischer Rechte zu anderen Ergebnissen führt als in den hochentwickelten Industrienationen, die die Verhandlungen über das TRIPS-Übereinkommen inhaltlich dominiert haben. Hier werden die Grenzen bei der Entwicklung einer globalen Wirtschafts- und Rechtsordnung sichtbar, bei der die unterschiedlichen technischen, wirtschaftlichen und sozialen Voraussetzungen der beteiligten Gesellschaften angemessene Berücksichtigung finden müssen.
\end{abstract}

